\title{
On the use of dynamic turbulence modelling in fire applications
}

\author{
Georgios Maragkos*, Bart Merci* \\ Department of Flow, Heat and Combustion Mechanics, Ghent University, St. \\ Pietersnieuwstraat 41, B-9000 Ghent, Belgium
}

\begin{abstract}
In an attempt to make a step forward towards predictive fire modelling, the application of turbulence modelling approaches, calculating the sub-grid scale viscosity, kinetic energy, turbulent Prandtl and Schmidt numbers based on a dynamic procedure, has been tested on a wide range of pool fire scenarios (i.e., MaCFP workshop test cases). Through the use of Large Eddy Simulations, it is investigated whether the applied modelling approaches can accurately predict the pool fire dynamics on different grid sizes and result in improved predictions compared to models using constant parameters. Additionally, it is evaluated whether the theoretical constants related to turbulence modelling, derived from isotropic decaying turbulence scenarios, are justified in turbulent buoyant flows.
\end{abstract}

Keywords: LES, FireFOAM, turbulence, dynamic Smagorinsky

\section{Introduction}

The use of Computation Fluid Dynamics (CFD) has been, and will continue to be for many years to come, an integral part of fire safety engineering. The use of CFD has allowed fire safety engineers to simulate and get insight into a variety of different fire scenarios which otherwise would be either too time consuming and expensive to conduct experimentally, or too complex to analyze with simple analytical solutions and zone models. Fire modelling has

\footnotetext{
*Corresponding author:

Email address: Georgios.Maragkos@UGent.be (Georgios Maragkos)
} 
been relying, to some extent, on models (e.g., Smagorinsky model [1], oneequation model [3], Vreman model [2]) whose coefficients have been derived for highly turbulent flows, conditions which are not truly representative of the ones encountered in turbulent buoyant fires. The use of dynamic models can help overcome such deficiencies but has not (yet) become the standard practice in fire modelling. The wide range of time and length scales, often encountered in typical fire scenarios, poses a significant problem in the application of such models mainly due to their increased computational cost. Nevertheless, the ever increasing hardware and software capabilities in computer science the past decades, has allowed more detailed and well-resolved CFD simulations for a wide range of engineering applications. It is expected that the use of dynamic modelling will be more feasible than ever in the near future, also for numerical simulations of (large-scale) fire applications.

In priniple, the predictive capabilities of dynamic turbulence models can be assumed to be superior, compared to models using constant coefficients, and particularly when it comes to fire modelling their advantages are multifold. Firstly, the laminar to turbulence transition, a key characteristic of all pool fires, can be captured without the need of tuning/adjusting the turbulence model parameters. Secondly, the sub-grid scale viscosity and kinetic energy evolve towards zero near walls without the explicit need for damping functions, which is a particularly useful feature since most fire scenarios involve either enclosures or some sort of surfaces. Thirdly, the predicted sub-grid scale (sgs) viscosity and kinetic energy do not explicitly depend on the grid size, since $\Delta$ is eliminated through the dynamic procedure (e.g., in the dynamic Smagorinsky when calculating $c_{s}$ for $\operatorname{sgs}$ viscosity and $c_{I}$ for sgs kinetic energy) and the ratio of the test filter to grid size is more relevant. Nevertheless, the filter width is still present in the calculation of the model parameters (e.g., $c_{s}$ and $c_{I}$ ). Finally, dynamic models will correctly tend towards zero sub-grid scale viscosity and kinetic energy as the DNS limit is reached, in contrast to constant coefficient models. Nevertheless, the dynamic models do also have their disadvantages, namely that they are computationally more expensive and can lead to oscillations and/or perform poorly on relatively coarse grids. The latter aspect is particularly important if these models are to be used for CFD of engineering purposes, hence, this aspect is explored within the present study. More in particular, simulations on relatively coarse grids are also employed in order to evaluate the potential of these dynamic models to accurately predict the characteristics of several pool fires. Focusing on the dynamic Smagorinsky model, the dyn- 
amic procedure employed to calculate the model parameters is well-known in the literature (e.g., $[4,5]$ ), as are the different variations of the model (e.g., standard, mixed $[6,7])$.

Within this framework, Large Eddy Simulations (LES), focusing mainly on turbulence modelling, are performed for a wide range for pool fire scenarios. The main objectives of this study are the following:

- Comprehensive application and evaluation of dynamic modelling approaches in numerical simulations of pool fires. Their accuracy and predictive capabilities are investigated over a wide range of grid sizes. Given its popularity within the scientific community, here the dynamic Smagorinsky model is employed for turbulence modelling even though the findings of the study are not limited to the specific model. Comparisons are also made to the constant Smagorinsky model in order to assess the advantages of the dynamic procedure when determining the turbulence model parameters.

- Evaluation whether the typically used constants related to turbulence modelling (i.e., $c_{s}$ for sgs viscosity, $c_{I}$ for sgs kinetic energy, $P r_{t}$ and $S c_{t}$ ), with their values derived from highly turbulent flows, are applicable / justified in turbulent buoyant flows.

Even though widely employed and validated for a great number of turbulent flow scenarios (e.g. [8, 9]), the usage of the dynamic Smagorinsky model is generally not standard practice for fire applications, with a few exceptions (e.g., $[10,11])$. The present work aims to cover part of this missing model validation in literature when it comes to fire scenarios. The current study is, to the authors' best knowledge, one of very few fire modelling studies in the context of LES where such an extensive usage of dynamic modelling has been employed and comprehensive validation has been performed. The usage of a dynamic procedure for determining the turbulent Schmidt, $S c_{t}$ and turbulent Prandtl, $\mathrm{Pr}_{t}$, numbers is also rather novel when it comes to fire scenarios. The research presented in this paper builds on the knowledge obtained by the authors in the past on fire plume modelling $[13,14,15,16,17]$ and aims at making a step forward towards predictive fire modelling.

It is important to note, however, that fully decoupling turbulence modelling from combustion and radiation modelling in the context of reacting buoyant plume applications is not feasible. Care should be taken when reporting numerical predictions in order to make sure that all the different physical 
aspects have been correctly modelled (i.e., there are no non-physical solutions) and to try to minimize any compensating effects that could influence the numerical predictions. The use of non-dissipative numerical schemes is important to this respect. Additionally, reporting of the radiative fractions, when these are predicted in the numerical simulations, is also essential for a better evaluation of the predicted flame temperatures. Overall, comparing both local and global quantities is of great interest when it comes to fire modelling since the combination of both give a much better indication of the accuracy of the numerical modelling employed in a numerical study. A stepwise approach is better suited for such evaluation purposes and therefore it is employed by the authors in this study. For this reason, a variety of test cases are considered where evaluation of all the different modelling approaches is performed, to the extent possible, separately.

\section{Modelling}

The CFD code fireFoam 2.2.x [18], originally developed by FM Global, is employed here. The code uses a fully compressible flow formulation and solves the Navier-Stokes equations, along with transport equations for species mass fractions and sensible enthalpy, using Favre-filtered quantities. The governing equations that the code employs are presented below:

$$
\begin{gathered}
\frac{\partial \bar{\rho}}{\partial t}+\nabla \cdot(\bar{\rho} \widetilde{u})=0 \\
\frac{\partial(\bar{\rho} \widetilde{u})}{\partial t}+\nabla \cdot(\bar{\rho} \widetilde{u} \widetilde{u})=-\nabla \bar{p}+\nabla \cdot\left[\mu_{e f f}\left(\nabla \widetilde{u}+(\nabla \widetilde{u})^{T}-\frac{2}{3}(\nabla \cdot \widetilde{u}) I\right)\right]+\bar{\rho} g \\
\frac{\partial\left(\bar{\rho} \widetilde{Y}_{k}\right)}{\partial t}+\nabla \cdot\left(\bar{\rho} \widetilde{u} \widetilde{Y}_{k}\right)=\nabla \cdot\left[\bar{\rho}\left(D_{k}+\frac{\nu_{s g s}}{S c_{t}}\right) \nabla \widetilde{Y}_{k}\right]+\overline{\dot{\omega}_{k}^{\prime \prime \prime}}, \quad\left(k=1, \ldots, N_{s}-1\right) \\
\frac{\partial\left(\bar{\rho} \widetilde{h}_{s}\right)}{\partial t}+\nabla \cdot\left(\bar{\rho} \widetilde{u} \widetilde{h}_{s}\right)=\frac{\overline{D p}}{D t}+\nabla \cdot\left[\bar{\rho}\left(D_{t h}+\frac{\nu_{s g s}}{P r_{t}}\right) \nabla \widetilde{h}_{s}\right]-\nabla \cdot \overline{\dot{q}_{r}^{\prime \prime}}+\overline{\dot{q}_{c}^{\prime \prime \prime}}
\end{gathered}
$$

where $\bar{\rho}$ is the density, $\widetilde{u}$ is the velocity vector, $\bar{p}$ is the pressure, $\mu_{e f f}=$ $\mu+\mu_{s g s}$ is the effective dynamic viscosity, $\mu$ is the laminar viscosity, $\mu_{s g s}$ is the sub-grid scale viscosity, $I$ is the identity tensor, $g$ is the gravitational acceleration, $\widetilde{Y}_{k}$ is the species mass fraction, $D_{k}$ is the species mass diffusivity, $S c_{t}$ is the turbulent Schmidt number, $\overline{\dot{\omega}}_{k}^{\prime \prime \prime}$ is the species reaction rate, $\widetilde{h}_{s}$ is the sensible energy, $D_{t h}$ is the thermal diffusivity, $P r_{t}$ is the turbulent Prandtl 
number, $\overline{\dot{q}_{r}^{\prime \prime}}$ is the radiative heat flux, $\overline{\dot{q}_{c}^{\prime \prime \prime}}=\Delta H_{c} \overline{\dot{\omega}_{F}^{\prime \prime \prime}}$ is the heat release rate per unit volume due to combustion and $\Delta H_{c}$ is the heat of combustion of the fuel. The code employs a unity Lewis assumption, effectively using the effective thermal diffusivity for the diffusion in both the species and sensible energy equations.

The dynamic Smagorinsky model [19] is used to model turbulence, calculating the sub-grid scale viscosity as:

$$
\mu_{\text {sgs }}=\bar{\rho}\left(c_{s} \Delta\right)^{2}|\widetilde{S}|
$$

where $\Delta$ is the filter width (taken as the cube root of the cell volume) and $\widetilde{S}$ is the (resolved) strain rate. The dynamic procedure employed for determining $c_{s}^{2}$ is summarized below:

$$
c_{s}^{2}=\frac{\frac{1}{2}\left\langle L_{i j} M_{i j}\right\rangle}{\left\langle M_{i j} M_{i j}\right\rangle}
$$

where the brackets denote averages which are computed as local averages of their face values. The Leonard term is defined as $L_{i j}=\widehat{\bar{\rho} \widetilde{u}_{i} \widetilde{u}_{j}}-\left(\widehat{\overline{\rho u_{i}}} \widehat{\rho u_{j}}\right) / \widehat{\bar{\rho}}$, $\beta_{i j}=-\widehat{\Delta}^{2} \widehat{\bar{\rho}}|\breve{\widetilde{S}}|\left(\breve{\widetilde{S}}_{i j}-\delta_{i j} \breve{\widetilde{S}}_{k k} / 3\right), \alpha_{i j}=-\Delta^{2} \bar{\rho}|\widetilde{S}|\left(\widetilde{S}_{i j}-\delta_{i j} \widetilde{S}_{k k} / 3\right)$ and $M_{i j}=$ $\beta_{i j}-\widehat{\alpha_{i j}}$. The hat denotes the application of a test filter while $\breve{\widetilde{f}}=\widehat{\overline{\rho f}} / \widehat{\bar{\rho}}$. The negative values of the Smagorinsky constant are clipped to zero to avoid potential numerical instabilities while no upper bound is defined for $c_{s}$. A simple top-hat filter was used as a test filter. Following [20], the ratio of the test filter to the LES filter is set to $\sqrt{6}$.

The sub-grid kinetic energy is estimated as [6]:

$$
k_{\text {sgs }}=c_{I} \Delta^{2}|\widetilde{S}|^{2}
$$

with the model parameter $c_{I}$ computed dynamically as:

$$
c_{I}=\frac{\left\langle\frac{1}{2} L_{k k} M_{k k}\right\rangle}{\left\langle M_{k k} M_{k k}\right\rangle}
$$

where the brackets denote averages, computed as local averages of their face values, with $L_{k k}=\widehat{\bar{\rho} \widetilde{u}_{k} \widetilde{u}_{k}}-\widehat{\bar{\rho} \widetilde{u}_{k} \bar{\rho} \widetilde{u}_{k}} / \overline{\bar{\rho}}$ and $M_{k k}=\widehat{\Delta}^{2} \widehat{\bar{\rho}}|\breve{S}|^{2}-\Delta^{2} \bar{\rho}|\widetilde{S}|^{2}$.

The sub-grid scale dissipation rate is modelled as [6]:

$$
\epsilon_{s g s}=\frac{c_{\epsilon} k_{s g s}^{3 / 2}}{\Delta}
$$


where $c_{\epsilon}=1.05[6]$ is a model constant. It is worth noting that tests using $\epsilon_{s g s}=\left(\mu_{\text {sgs }} / \rho\right)|\widetilde{S}|^{2}$ to model the sub-grid scale dissipation rate were not successful due to the zero values the sub-grid scale viscosity obtained in the near-field region of fire plumes (i.e., laminar regions). In addition, a dynamic procedure [21] for calculating $c_{\epsilon}$ was also tested without success due to the very low values the $c_{\epsilon}$ obtained just above the fire source, which strongly affect the fuel reaction rates.

The turbulent Prandtl number is computed dynamically as [7]:

$$
P r_{t}=\frac{c_{s}^{2}\left\langle T_{j} T_{j}\right\rangle}{\left\langle K_{j} T_{j}\right\rangle}
$$

where $T_{j}=-\widehat{\Delta}^{2} \widehat{\bar{\rho}}|\breve{\widetilde{S}}| \frac{\partial \breve{T}}{\partial x_{j}}+\Delta^{2} \bar{\rho}|\widetilde{S}| \frac{\partial \widetilde{T}}{\partial x_{j}}$ and $K_{j}=\left(\frac{\widehat{\overline{\rho u_{j}} \overline{\rho T}}}{\bar{\rho}}-\frac{\widehat{\widehat{\rho u_{j}}} \widehat{\widehat{\rho T}}}{\widehat{\bar{\rho}}}\right)$. The $\operatorname{Pr}_{t}$ values are clipped between 0.05 and 1.0 to ensure numerical stability.

The turbulent Schmidt number is computed dynamically as [7]:

$$
S c_{t}=\frac{c_{s}^{2}\left\langle M_{j} M_{j}\right\rangle}{\left\langle L_{j} M_{j}\right\rangle}
$$

where $M_{j}=-\widehat{\Delta}^{2} \widehat{\bar{\rho}}|\breve{S}| \frac{\partial \breve{Y}_{k}}{\partial x_{j}}+\Delta^{2} \bar{\rho}|\widetilde{S}| \frac{\partial \widetilde{Y}_{k}}{\partial x_{j}}$ and $L_{j}=\left(\frac{\widehat{\rho u_{j} \overline{\rho Y_{k}}}}{\bar{\rho}}-\frac{\widehat{\rho u_{j}} \widehat{\widehat{\rho Y_{k}}}}{\widehat{\bar{\rho}}}\right)$. The $S c_{t}$ values are clipped between 0.05 and 1.0 to ensure numerical stability. The dynamic model for the turbulent Schmidt number has only been employed in the non-reacting case considered here (i.e., Sandia helium plume case), since the case is isothermal and the dynamic turbulent Prandtl number model could not be used. For all the reacting cases, the assumption of $S c_{t} \approx P r_{t}$ has been employed, effectively only using the dynamic $P r_{t}$ model. The subgrid scale thermal diffusivity is calculated as $\alpha_{s g s}=\mu_{s g s} / P r_{t}$.

The combustion model considered employs a one-step, infinitely fast, irreversible chemical reaction for the fuel combined with the Eddy Dissipation Model (EDM) [22] to deal with turbulence-chemistry interactions. Within the EDM model the fuel mass reaction rate is calculated as:

$$
\overline{\dot{\omega}_{F}^{\prime \prime \prime}}=\bar{\rho} \frac{\min \left(\widetilde{Y}_{F}, \widetilde{Y}_{O_{2}} / s\right)}{\tau_{m i x}}
$$

where $\widetilde{Y}_{F}$ and $\widetilde{Y}_{O_{2}}$ are the (filtered) fuel and oxygen mass fractions, respectively and $s$ is the stoichiometric oxygen-to-fuel mass ratio. The $\overline{\dot{\omega}_{k}^{\prime \prime \prime}}$ for the others species are obtained through simple stoichiometric relations. 
The mixing time scale is calculated as:

$$
\tau_{\text {mix }}=\frac{k_{\text {sgs }}}{A \epsilon_{s g s}}=\frac{1}{A c_{\epsilon} \sqrt{c_{I}} \widetilde{S}}
$$

where the model parameter has been assigned the value $A=2$, which corresponds to an average value across the flame brush based on the theoretical analysis of Brizuela \& Bilger [23]. It is worth noting that with the current formulation the fuel reaction rate is independent of the grid size, $\Delta$, a user-defined parameter which is rather artificial. The EDM combustion model essentially considers that chemical reactions are infinitely fast and that combustion is governed by mixing, hence, the turbulent mixing time is the dominant time scale used in the calculation of the fuel reaction rate. It is a simple and robust model which can consider whether the mixture is lean or rich, but cannot be directly employed in scenarios where finite rate chemistry effects might be important (e.g., NO formation, local extinction).

The radiation modelling approach employed considers the radiative intensity to be a function of both spatial location and angular direction and is obtained by solving the radiative transfer equation (RTE) using the finite volume discrete ordinates model (fvDOM). The absorption/emission is modelled through the weighted-sum-of-gray-gases model (WSGGM). Within WSGGM, the total emissivity and absorptivity (assuming $\alpha=\epsilon$ ) of a gas mixture is calculated as the sum of fictitious gray gases, weighted with a temperature dependent weighting factor as:

$$
\widetilde{\epsilon}=\sum_{i=0}^{I} \widetilde{a}_{\epsilon, i}(T)\left(1-e^{-\kappa_{i} p L}\right)
$$

where $a_{\epsilon, i}$ is the emissivity weight factor of the $i-t h$ fictitious gray gas, $\kappa_{i}$ is the absorption coefficient of the $i-t h$ gray gas, $p$ is the sum of the partial pressures of all absorbing gases and $L$ is the path length, calculated as $L=3.6 \mathrm{~V} / \mathrm{A}$. The volume, $V$, is calculated by summing all the cell volumes where reaction takes place. Assuming a certain flame shape (e.g., rectangular or conical depending on the fuel source configuration), the corresponding surface area, $A$, can be calculated. This approach effectively calculates the path length dynamically during the simulations, as opposed to fixing it to a constant value. The temperature dependence of $a_{\epsilon, i}$ is calculated as:

$$
\widetilde{a}_{\epsilon, i}=\sum_{i=0}^{J} b_{\epsilon, i, j} \widetilde{T}^{j-1}
$$


where $b_{\epsilon, i, j}$ are the emissivity gas temperature polynomial coefficients. The coefficients $b_{\epsilon, i, j}$ and $\kappa_{i}$ are taken from [24]. The total absorptivity is then calculated as:

$$
\widetilde{a}=-\frac{\ln (1-\widetilde{\epsilon})}{L}
$$

and the radiative heat fluxes in Equation (4) are calculated as:

$$
\nabla \cdot \overline{\dot{q}_{r}^{\prime \prime}}=\widetilde{a}\left(4 \sigma \widetilde{T}^{4}-\widetilde{G}\right)
$$

where $\sigma$ is the Stefan-Boltzmann constant and $G$ is the total irradiance.

In addition to the WSGGM model, a simpler approach for radiation modelling has also been considered, which assumes a non-absorbing, optically thin medium. Subsequently, the radiative source term in Equation (4) can also be calculated as:

$$
\nabla \cdot \overline{\dot{q}_{r}^{\prime \prime}}=\chi_{r} \overline{\dot{q}_{c}^{\prime \prime \prime}}
$$

where $\chi_{r}$ is a constant global radiative fraction for the fuel. This approach has only been considered in the numerical simulations for the McCaffrey fire plumes. The motivation behind this choice is to guarantee the correct amount of heat being released due to radiation so that the evaluation of the combustion model can be done independently of any uncertainties related to simultaneously trying to predict the radiative fractions.

\section{Experimental test cases}

The experiments considered here for validation purposes are all test cases of the MaCFP workshop [12] (https://iafss.org/macfp/) and were chosen by the authors since they have been identified by the fire community as the 'best' cases, at least to date, for CFD validation. A detailed description of the different test cases is already available (https://github.com/MaCFP), hence, only a brief overview is outlined here.

All the cases considered here are turbulent, hence, relevant to study the effect of turbulence modelling in fire scenarios. The helium plume case mimics a medium/large scale fire without the added complexity of modelling combustion and radiation. The effect of turbulence on the predicted velocities, helium mass fractions and puffing frequencies can be examined. The McCaffrey test case is useful for determining whether, with the chosen modelling options, there is correct scaling of the temperature and velocity with the heat release rate of the fire. The UMD line burner case consists of a fairly 
new database where experimental data of centerline and radial temperatures are available with fairly small experimental uncertainties. In this case, the effect of turbulence is assessed by comparing both first and second order statistics of temperature. The methanol pool fire is a small/medium scale fire and is essentially a suitable test case, in terms of available experimental data for model validation (first and second order statistics of velocities and temperatures at different heights). Overall, the experiments considered involve buoyant plumes with small / large scale turbulence, non-reacting / reacting plumes of different fuels and diameters, the combination of which enables the study of turbulence modelling in the context of fire applications and provides a comprehensive set of experimental data for validation purposes.

\subsection{Sandia helium plume}

Sandia's helium plume experiments were performed in the FLAME facility and reported by OHern et al. [25]. The FLAME chamber consists of a $6.1 \mathrm{~m}$ cubical enclosure with a $2.4 \mathrm{~m}$ in diameter chimney located on top of the chamber. The experiment was designed to be a canonical buoyant plume so that the results would not be dependent on the specific experimental geometry. The plume source is $1 \mathrm{~m}$ in diameter, surrounded by a $0.5 \mathrm{~m}$ wide floor, issued from a diffuser at an average velocity of $0.325 \mathrm{~m} / \mathrm{s}$. The inlet mixture contained $96.4 \%$ helium $(\mathrm{He}), 1.7 \%$ acetone $\left(\mathrm{C}_{3} \mathrm{H}_{6} \mathrm{O}\right)$ and $1.9 \%$ oxygen $\left(\mathrm{O}_{2}\right)$ by volume with a molecular weight of $5.45 \mathrm{~g} / \mathrm{mol}$. The reported experimental uncertainty on the measured velocities and turbulent statistics is in the order of $20 \%$ and $30 \%$, respectively, while for the mean and rms mass fractions is in the order of $23 \%$ and $21 \%$, respectively.

\subsection{McCaffrey's fire plumes}

McCaffrey's experiments [26] consisted of a $30 \mathrm{~cm}$ square burner using natural gas (45 MJ/kg assuming $19 \mathrm{~kg} / \mathrm{kgmol}$ as mole weight) as fuel. Experiments with the burner raised $75 \mathrm{~cm}$ from the ground or flushed to the floor were performed with resulting heat release rates of $14.4 \mathrm{~kW}, 21.7 \mathrm{~kW}, 33$ $\mathrm{kW}, 44.9 \mathrm{~kW}$ and $57.5 \mathrm{~kW}$. The interesting feature of these experiments was that the experimental data of centerline temperature and velocity collapsed when scaled by the Froude number.

\subsection{UMD line burner}

The experiments consist of a low-strain, buoyancy-driven, fully-turbulent diffusion flame in a canonical line-fire configuration reported by White et al. 
[27]. The fuel mass flow rate (i.e., methane of purity $99.5 \%$ ) was set to $1 \mathrm{~g} / \mathrm{s}$ while the co-flowing oxidizer (i.e., air) was provided at $85 \mathrm{~g} / \mathrm{s}$, according to the experiments. Assuming complete combustion the resulting heat release rate was approximately $50 \mathrm{~kW}$.

\subsection{Waterloo methanol pool fire}

Medium-scale, $30.5 \mathrm{~cm}$ in diameter, methanol pool fire experiments were reported by Weckman et al. [28]. The methanol fuel feed rate was maintained at $1.35 \mathrm{~cm}^{3} / \mathrm{s}$ to maintain a burner rim height of $1 \mathrm{~cm}$. Assuming complete combustion, the total heat release rate was approximately $21.3 \mathrm{~kW}$. An experimental uncertainty of $5 \%$ was reported for the mean and rms values of velocity and mean values of temperature while $30 \%$ errors were estimated for the rms temperatures.

\section{Numerical setup}

An overview of the different computational domains, grid sizes, total number of cells, the most notable initial and boundary conditions, total running times, averaging periods, time steps and models employed for simulating the different cases is outlined in Table 1. For all the cases considered in this study, a local refinement strategy is employed in order to have a good grid resolution in the near-field region of the plumes (i.e., covering axial locations up to the experimentally reported flame heights). The choice of grid sizes used to simulate the different test cases in this study was made to be in the order of $1 \mathrm{~cm}$ or less, based on what has been reported in [12], while also

making sure that the ratio of the characteristic fire size, $D^{*}=\left(\frac{\dot{Q}}{\rho_{\infty} c_{p} T_{\infty} \sqrt{g}}\right)^{2 / 5}$, to the grid size, $\Delta$, was higher than 10 [29]. The mass flow rate, $\dot{m}(\mathrm{~kg} / \mathrm{s})$, is applied at the fuel source (accounting for both convective and diffusive mass fluxes), calculated based on the experimentally reported values. The equations are advanced in time using a second order backward scheme. For the convective terms a second order filtered linear scheme (i.e., filteredLinear2V) is employed while for scalar transport a TVD scheme (i.e., limitedLinear) which guarantees that the values for the species mass fractions remain between 0 and 1 . For the diffusive terms a central difference scheme is used. For the solution of the Radiative Transfer Equation (RTE), 48 solid angles are used for angular discretization. A PIMPLE algorithm is used for the pressure-velocity coupling with a Rhie-Chow interpolation to avoid oddeven decoupling. A mixed boundary condition is assigned for velocity at the 
Table 1: Overview of the setup used in the numerical simulations.

\begin{tabular}{|c|c|c|c|c|}
\hline Case & $\begin{array}{l}\text { Sandia helium } \\
\text { plume [25] }\end{array}$ & $\begin{array}{l}\text { McCaffrey's } \\
\text { fire plumes [26] }\end{array}$ & $\begin{array}{l}\text { UMD line } \\
\text { burner }[27]\end{array}$ & $\begin{array}{c}\text { Waterloo methanol } \\
\text { pool fire }[28]\end{array}$ \\
\hline Domain & $\begin{array}{l}4 \mathrm{~m} \times 4 \mathrm{~m} \\
\text { (cylidrical) }\end{array}$ & $\begin{array}{c}3 \mathrm{~m} \times 3.3^{f} \mathrm{~m} \times 3 \mathrm{~m} \\
\text { (rectangular) }\end{array}$ & $\begin{array}{c}1.6 \mathrm{~m} \times 2 \mathrm{~m} \times 1 \mathrm{~m} \\
\text { (rectangular) }\end{array}$ & $\begin{array}{l}1.5 \mathrm{~m} \times 1.8 \mathrm{~m} \\
\text { (cylidrical) }\end{array}$ \\
\hline Fuel source & $\begin{array}{l}1 \text { m diameter }^{d} \\
\text { (circular) }\end{array}$ & $\begin{array}{c}0.3 \mathrm{~m} \times 0.3 \mathrm{~m} \\
\text { (square) }\end{array}$ & $\begin{array}{c}0.5 \mathrm{~m} \times 0.05 \mathrm{~m} \\
\text { (line) }\end{array}$ & $\begin{array}{l}0.305 \mathrm{~m} \text { diameter } \\
\text { (circular) }\end{array}$ \\
\hline Fuel & $\left(\mathrm{He}, \mathrm{C}_{3} \mathrm{H}_{6} \mathrm{O}, \mathrm{O}_{2}\right)^{e}$ & $\mathrm{CH}_{4}$ & $\mathrm{CH}_{4}$ & $\mathrm{CH}_{3} \mathrm{OH}$ \\
\hline Grid size $(\Delta)^{a}$ & $\begin{array}{c}1.5 \mathrm{~cm} \\
(1.5 \mathrm{~m} \times 1 \mathrm{~m}) \\
3 \mathrm{~cm} \\
(2 \mathrm{~m} \times 2 \mathrm{~m}) \\
6 \mathrm{~cm} \\
\text { (domain) }\end{array}$ & $\begin{array}{c}1.5 \mathrm{~cm} \\
\left(0.9 \mathrm{~m} \times 1.8^{f} \mathrm{~m} \times 0.9 \mathrm{~m}\right) \\
3 \mathrm{~cm} \\
\left(1.5 \mathrm{~m} \times 3.3^{f} \mathrm{~m} \times 1.5 \mathrm{~m}\right) \\
6 \mathrm{~cm} \\
\text { (domain) }\end{array}$ & $\begin{array}{c}0.625 \mathrm{~cm} \\
(0.6 \mathrm{~m} \mathrm{x} 0.6 \mathrm{~m} \times 0.4 \mathrm{~m}) \\
1.25 \mathrm{~cm} \\
(0.8 \mathrm{~m} \mathrm{x} 0.8 \mathrm{~m} \mathrm{x} 0.6 \mathrm{~m}) \\
2.5 \mathrm{~cm}^{h} \\
(\text { domain })\end{array}$ & $\begin{array}{c}0.5 \mathrm{~cm} \\
(0.6 \mathrm{~m} \mathrm{x} 0.6 \mathrm{~m}) \\
1 \mathrm{~cm} \\
(0.9 \mathrm{~m} \mathrm{x} 0.9 \mathrm{~m}) \\
2 \mathrm{~cm}^{i} \\
\text { (domain) }\end{array}$ \\
\hline$D^{*} / \Delta^{b}$ & - & $12-20$ & see notes & 42 \\
\hline Cells $^{c}$ & 0.44 million & 0.945 million & 0.886 million & 0.655 million \\
\hline $\begin{array}{l}\text { Initial } \\
\text { conditions }\end{array}$ & $\begin{array}{c}T_{\infty}=285 \mathrm{~K} \\
P_{\infty}=80900 \mathrm{~Pa}\end{array}$ & $\begin{array}{c}T_{\infty}=293 \mathrm{~K} \\
P_{\infty}=101325 \mathrm{~Pa}\end{array}$ & $\begin{array}{c}T_{\infty}=293 \mathrm{~K} \\
P_{\infty}=101325 \mathrm{~Pa}\end{array}$ & $\begin{array}{c}T_{\infty}=293 \mathrm{~K} \\
P_{\infty}=101325 \mathrm{~Pa}\end{array}$ \\
\hline $\begin{array}{l}\text { Boundary } \\
\text { conditions }\end{array}$ & $\begin{array}{c}T_{\text {inlet }}=285 \mathrm{~K} \\
\dot{m}=47.4773 \mathrm{~g} / \mathrm{s}\end{array}$ & $\begin{aligned} & T_{\text {inlet }}=293 \mathrm{~K} \\
\dot{m}= & 0.2878-1.1493 \mathrm{~g} / \mathrm{s}\end{aligned}$ & $\begin{array}{c}T_{\text {inlet }}=293 \mathrm{~K} \\
\dot{m}=1 \mathrm{~g} / \mathrm{s}\end{array}$ & $\begin{array}{l}T_{\text {inlet }}=338^{k} \mathrm{~K} \\
\dot{m}=1.0692 \mathrm{~g} / \mathrm{s}\end{array}$ \\
\hline $\operatorname{HRR}(\dot{Q})$ & - & $14.4 \mathrm{~kW}-57.5 \mathrm{~kW}$ & $50 \mathrm{~kW}$ & $21.3 \mathrm{~kW}$ \\
\hline Run time & $35 \mathrm{~s}$ & $35 \mathrm{~s}$ & $35 \mathrm{~s}$ & $35 \mathrm{~s}$ \\
\hline Aver. time & $30 \mathrm{~s}$ & $30 \mathrm{~s}$ & $30 \mathrm{~s}$ & $30 \mathrm{~s}$ \\
\hline Time step & $C o=0.9$ & $C o=0.9$ & $C o=0.9$ & $C o=0.9$ \\
\hline $\begin{array}{l}\text { Turb. model } \\
\text { Dyn. parameters }\end{array}$ & $\begin{array}{l}\text { Dyn. Smag. } \\
c_{s}, S c_{t}\end{array}$ & $\begin{array}{l}\text { Dyn. Smag. } \\
c_{s}, c_{I}, P r_{t}\end{array}$ & $\begin{array}{l}\text { Dyn. Smag. } \\
c_{s}, c_{I}, P r_{t}\end{array}$ & $\begin{array}{l}\text { Dyn. Smag. } \\
c_{s}, c_{I}, P r_{t}\end{array}$ \\
\hline Comb. model & - & $\operatorname{EDM}(\mathrm{A}=2)$ & $\operatorname{EDM}(\mathrm{A}=2)$ & $\operatorname{EDM}(\mathrm{A}=2)$ \\
\hline Rad. model & $\begin{array}{l}- \\
-\end{array}$ & $\begin{array}{l}\text { fvDOM } \\
\text { const. rad. fraction }\end{array}$ & $\begin{array}{l}\text { fvDOM } \\
\text { WSGGM }\end{array}$ & $\begin{array}{l}\text { fvDOM } \\
\text { WSGGM }\end{array}$ \\
\hline
\end{tabular}

${ }^{a}$ The parentheses indicate the areas where the local grid refinement with the corresponding grid size has been used.

${ }^{b}$ Based on the finest grid size employed. The characteristic fire diameter is defined as $D^{*}=\left(\frac{\dot{Q}}{\rho_{\infty} c_{p} T_{\infty} \sqrt{g}}\right)^{2 / 5}$. Note that the ratio is perhaps not so relevant for the UMD line burner case given the burner geometry (i.e., very small width compared to its length).

${ }^{c}$ Corresponds to the total number of cells with the finest grid size employed.

${ }^{d}$ The fuel source is surrounded by a $0.5 \mathrm{~m}$ wide floor.

${ }^{e}$ Mixture containing $96.4 \%$ helium $(\mathrm{He}), 1.7 \%$ acetone $\left(\mathrm{C}_{3} \mathrm{H}_{6} \mathrm{O}\right)$ and $1.9 \%$ oxygen $\left(\mathrm{O}_{2}\right)$ by volume. The molecular weight of the mixture was $5.45 \mathrm{~g} / \mathrm{mol}$ and was issued from the diffuser at an average velocity of $0.325 \mathrm{~m} / \mathrm{s}$. A molecular Schmidt number of 0.2 has been used for He while 0.7 for the other species.

${ }^{f}$ The computational domain extends in the axial direction from $\mathrm{y}=-0.3 \mathrm{~m}$ up to the corresponding axial height with the burner located at $\mathrm{y}=0 \mathrm{~m}$ (i.e., burner raised $30 \mathrm{~cm}$ from the bottom boundary). The local mesh refinement starts from $\mathrm{y}=-0.3 \mathrm{~m}$ up to the corresponding axial height.

${ }^{g}$ Prescribed constant radiative fractions of $0.17,0.21,0.25,0.27,0.27$ for the $14.4 \mathrm{~kW}, 21.7 \mathrm{~kW}, 33 \mathrm{~kW}, 44.9 \mathrm{~kW}, 57.5 \mathrm{~kW}$, respectively, based on the experimental measurements [30].

${ }^{h}$ Stretched in the axial direction with a ratio of final to initial cell length of 1.5 .

${ }^{i}$ Stretched in the axial and radial directions with a ratio of final to initial cell length of 1.5.

${ }^{j}$ The fuel source is surrounded by a rim which is $1 \mathrm{~cm}$ in height. In the $2 \mathrm{~cm}$ grid size case the height of the rim is $2 \mathrm{~cm}$.

${ }^{k}$ Corresponds to the evaporation temperature of $\mathrm{CH}_{3} \mathrm{OH}$. 
open boundaries of the computational domain, setting zero gradient for any outward flow and calculating the inlet velocity from pressure. A Dirichlet boundary condition is assigned for pressure at the open boundaries of the computational domain, which fixes total pressure, and when velocity changes, the pressure is adjusted accordingly. At the outlet (i.e., top boundary), a Neumann (i.e., zero gradient) boundary condition is assigned for all variables with no reverse flow allowed in order to avoid numerical instabilities. At the walls, a no-slip boundary condition is used for velocity, a small value (i.e., $10^{-6}$ ) is assigned for the sub-grid scale viscosity and kinetic energy while zero gradient is employed for the chemical species.

\section{Results}

First an evaluation of the predicted turbulence modelling parameters is presented, followed by a comprehensive evaluation of the dynamic modelling approaches by comparing the numerical predictions to experimental data. The reported temperature results are thermocouple temperatures obtained from a simple thermal balance for the thermocouple bead (treated as a thermally-thin device) as described in [31].

\subsection{Turbulence modelling parameters}

A dynamic procedure was employed to determine the various turbulence modelling parameters employed in the current study. These parameters were $c_{s}$ (used for determining the sub-grid scale dynamic viscosity), $c_{I}$ (used for determining the sub-grid scale kinetic energy) and $S c_{t} / P r_{t}$ (used for calculating the turbulent mass diffusion and turbulent thermal diffusion fluxes, respectively). The theoretical values for $c_{s}$ and $c_{I}$ are approximately 0.17 [41] - 0.2 [42] and 0.18 [6] - 0.202 [42], respectively, while values in the order of 0.5-0.7 are typically used for $S c_{t} / P r_{t}$ for turbulent buoyant flows.

As a first step, and in order to verify the implementation of the models, comparisons are made to the low Reynolds number data of ComteBellot and Corrsin [44]. A periodic box of side $L=9 \times 2 \pi(\approx 0.566 \mathrm{~m})$ and $\nu=1.5 \times 10^{-5} \mathrm{~m}^{2} / \mathrm{s}$ for kinematic viscosity is used. The initial condition for the simulation was generated by superimposing Fourier modes with random phases such that the spectrum matched that of the initial experimental data [45], and taken from [46]. The experiment consists of a flow which is unfor-

ced, so that the decay of the total kinetic energy in time is due to viscous dissipation. Overall, the agreement between the numerical simulations and 


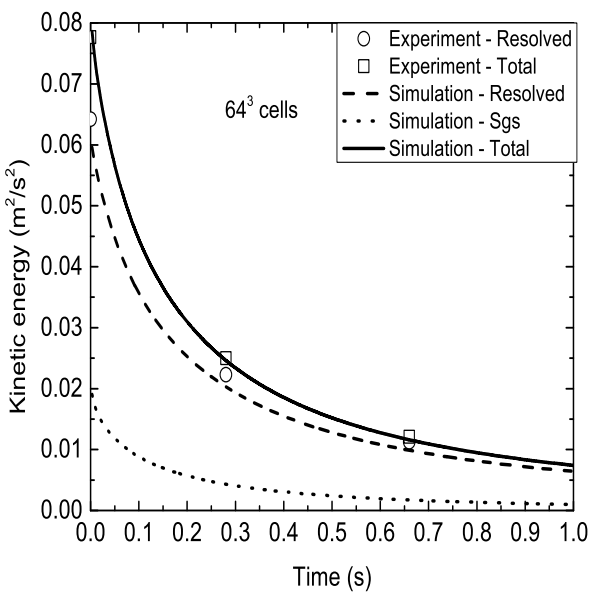

(a)

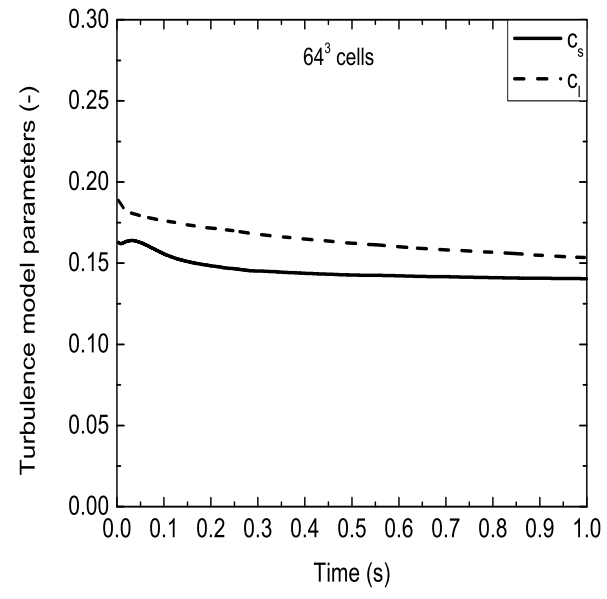

(b)

Figure 1: Time history of (a) kinetic energy and (b) turbulence model parameters for a grid resolution of $64^{3}$.

the experimental data, shown in Figure 1(a), is satisfactory. The resulting values for the $c_{s}$ and $c_{I}$ parameters from the numerical simulations, presented in Figure 1(b), are in the order of 0.15-0.16 and 0.17-0.18, respectively, close to the theoretical values suggested in the literature.

The predicted values of the turbulence modelling parameters from the numerical simulations of the different tests cases are presented in Figure 2 in order to evaluate whether their theoretical values, previously obtained from the isotropic decaying turbulence scenario, can be justified in typical pool fire scenarios. It should be noted that in the centerline results the axial distance, $y$, has been normalized by the equivalent flame height, $L_{f}$, calculated based on the $97 \%$ heat release rate criterion. Results for the Sandia helium plume are also shown with the axial distance normalized by the location where the mean He mass fraction was $20 \%$ the value of the inlet. It is observed that $c_{s}$ and $c_{I}$ are significantly lower in the near-field region of the fire plumes (i.e., $y / L_{f}<0.1$ ), with values starting from close to zero and increasing with axial distance away from the fire source. This indicates that the laminar region, often found at the base of most fire plume scenarios, can be captured by the models. This aspect is particularly important for accurately predicting the laminar to turbulence transition in pool fires. The average values further downstream are in the order of 0.1 and 0.15 , respectively, which is still significantly lower than the theoretical values of approximately 0.17 and 0.2 

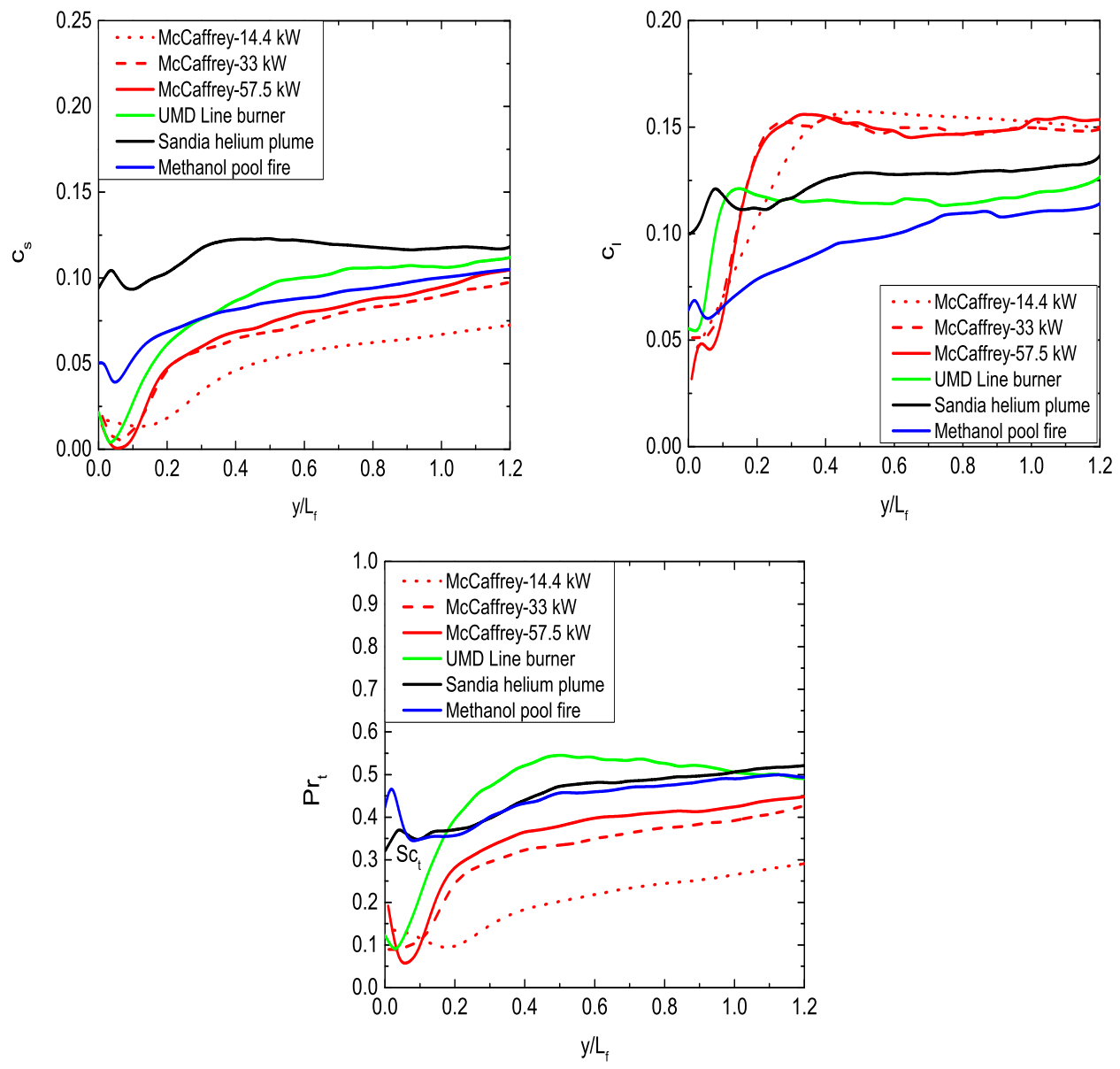

(c)

Figure 2: Predicted centerline values of (a) $c_{s}$, (b) $c_{I}$ and (c) $P r_{t}$ with the finest grid sizes employed in the simulations.

for isotropic decaying turbulence. The $c_{s}$ values for the Sandia helium plume start from approximately 0.1 at the fuel source and increase with axial distance up to approximately 0.12 . The fact that $c_{s}$ does not start from very low values (i.e., close to zero like in the fire plume cases) indicates that some turbulence is present at the fuel source. This is indeed verified since the calculated Reynolds number, based on the inlet conditions, is in the order of 3200 which would correspond to an internal flow in the transition regime. Significantly low values (i.e., in the order of 0.1-0.2) are predicted for $\operatorname{Pr}_{t}$ 
just above the fire source for most of the scenarios examined, with the values increasing (i.e., close to 0.5) further downstream. A variation in the turbulent Schmidt number, $S c_{t}$, starting from 0.3, close to the fuel inlet, up to 0.5, further downstream, is also evident for the helium plume case. It is clear that the use of a constant values for all the above-mentioned parameters cannot accurately capture the significant spatial variation observed above the pool fire source. Turbulent Prandtl and Schmidt number values from DNS simulations of thermal plumes have been reported in the literature in the past and are, overall, in the same order of magnitude as the values reported in our study. According to Eidson [47] the typical range of $P r_{t}$ lies between $1 / 3$ and $1 / 2$. Craskel et al. [48] reported that $\operatorname{Pr}_{t}$ in a pure plume is $3 / 5$ when the the mean profiles of velocity and buoyancy have a Gaussian form of equal width. DNS data on pure thermal plumes, reported by Pham et al. [49], exhibited values in the order of $P r_{t} \approx 0.2$ and $c_{s} \approx 0.1$ just above the plume source with increasing tendency away from the inlet. Experimental $\mathrm{Pr}_{t}$ values ranging from 0.05 - 0.5 [28] have been reported above the fire source of the methanol pool fire considered here. Based on scaling analysis, the radial-mean turbulent Schmidt number was reported to be about 0.75 in the near field of a $1 \mathrm{~m}$ He plume [50]. Additional DNS and experimental data reporting on the local variation of $P r_{t}$ and $S c_{t}$ in the near field-region of buoyant plumes would be helpful for further model validation/evaluation. Nevertheless, since fluid transport in the resolved scales of most buoyant plumes is mainly advection (i.e., not diffusion) dominated it is expected that the relative influence of $P r_{t}$ and $S c_{t}$ will be smaller than that of $c_{s}$.

\subsection{Sandia helium plume}

Results for the predicted mean and rms values of helium mass fractions, axial and radial velocities are presented in Figures 3-4. The experimental data are depicted with symbols while the gray-shaded areas represent the experimental uncertainty. It should be noted that considering the exact helium/acetone/oxygen composition at the plume inlet is a pre-requisite for accurate numerical predictions of both the mean and rms helium mass fractions. Overall, very good predictions are obtained for both the mean and rms quantities on all the different grid sizes used with the numerical simulations being, in most cases, within the experimental uncertainty. The increase in axial velocity at locations further downstream, due to buoyancy, and the high radial velocities close to the plume source, due to air entrainment, are accurately captured by the simulations. Some small discrepancies are pre- 
sent, with the most notable being the over-predicted mean and rms helium mass fractions at height $y=0.6 \mathrm{~m}$. It is believed that the use of even finer grid size would eventually further improve the predictions at this location.

A fast Fourier transformation on the instantaneous signal of the axial velocity on the centerline at height $y=0.5 \mathrm{~m}$ has been performed. This specific location was chosen to be the same as in the experiments. The experimental puffing frequency is reported to be $1.37 \pm 0.1 \mathrm{~Hz}$. The experimental correlation, $f=0.8 R i^{0.38} U / D$, suggested by Cetegen et al. [32] for $R i<100$, leads to a puffing frequency of $1.34 \mathrm{~Hz}$, while the established puffing frequency correlation for buoyant diffusion flames of various fuels by Cetegen et al. [33], $f=1.5 / \sqrt{D}$, yields a frequency of $1.5 \mathrm{~Hz}$, independent of flow conditions. Based on Figure 5, the predicted puffing frequencies from the numerical simulations with grid sizes of $1.5 \mathrm{~cm}, 3 \mathrm{~cm}$ and $6 \mathrm{~cm}$ are 1.46 $\mathrm{Hz}, 1.43 \mathrm{~Hz}$ and $1.53 \mathrm{~Hz}$, respectively, all of them well within the range of expected puffing frequencies based on the above-reported experimental correlations. Nevertheless, the predicted peak for the case with a $6 \mathrm{~cm}$ grid size is not as distinct as in the finer grid size cases. Interestingly, the decay of the energy spectrum has a slope which is close to $-5 / 3$ for all the grid sizes.

\subsection{McCaffrey fire plumes}

From McCaffrey's experimental data, if the downstream distance, $y$, away from the fire source is scaled with $\dot{Q}^{2 / 5}$, the sets of data for the centerline temperature rise and the centerline velocity normalized by $\dot{Q}^{1 / 5}$, collapse onto an almost single curve, independent of the heat release rate. McCaffrey's correlations, presented as dashed black lines, are used in this section to evaluate the numerical predictions, presented in Figure 6.

The numerical predictions with grid sizes of $1.5 \mathrm{~cm}$ and $3 \mathrm{~cm}$ are very satisfactory and reproduce, reasonably well, the scaling laws as suggested by McCaffrey. As expected, the results with the finest grid size employed (i.e., $1.5 \mathrm{~cm}$ ) are qualitatively better in terms of both data clustering and changing of slopes. It should be noted that the actual experimental data for temperature rise in the persistent flame region, $y / \dot{Q}^{2 / 5}<0.08$, do exhibit a decrease similar to what the simulations predict on the $1.5 \mathrm{~cm}$ grid size. This aspect is not evident when using the experimental correlation for comparison purposes. The results on a $6 \mathrm{~cm}$ grid size are poor, severely under-predicting both the centerline temperatures and axial velocities for all heat release rates. This finding is perhaps not surprising as this case corresponds to only 5 grid 

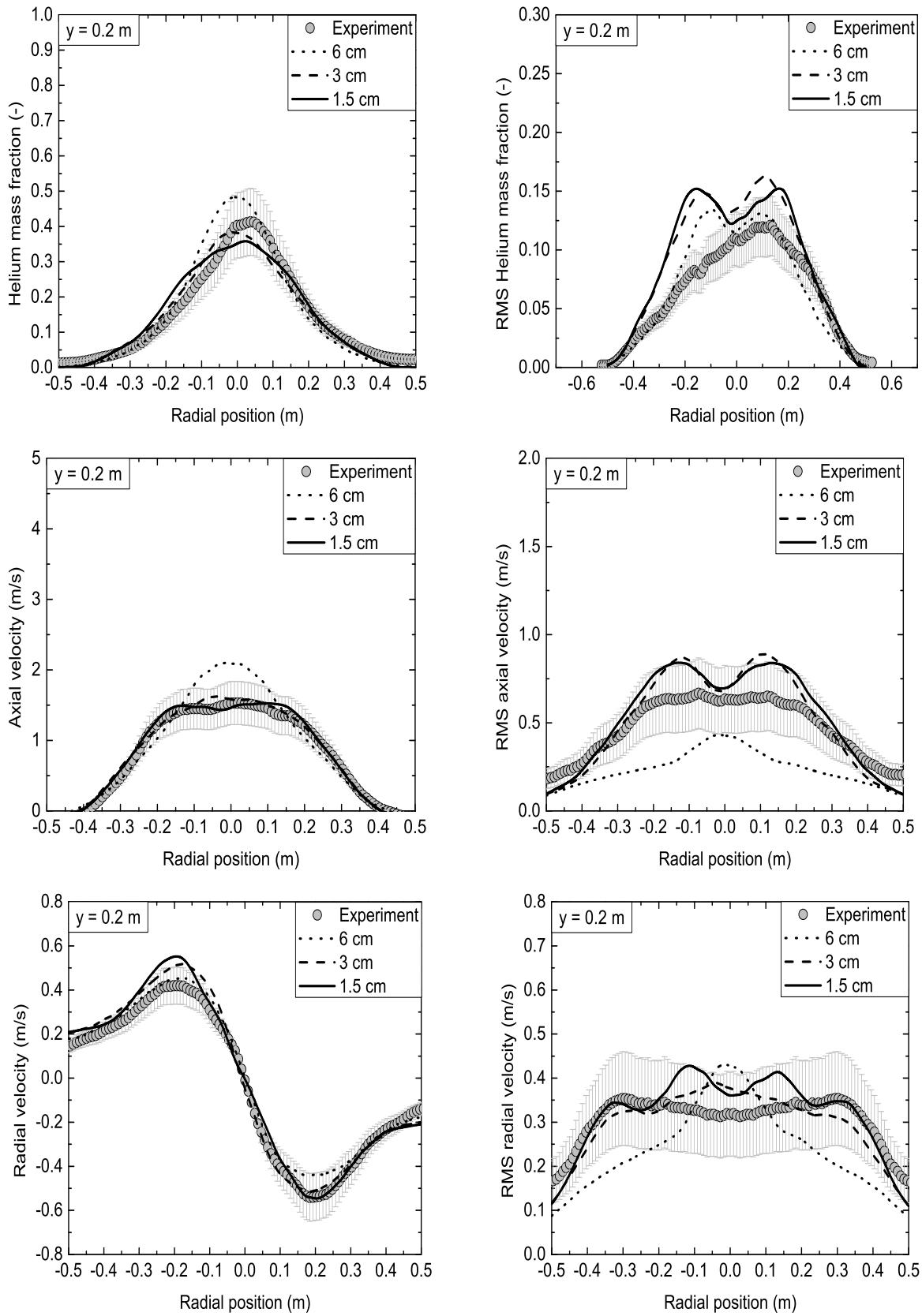

(a)

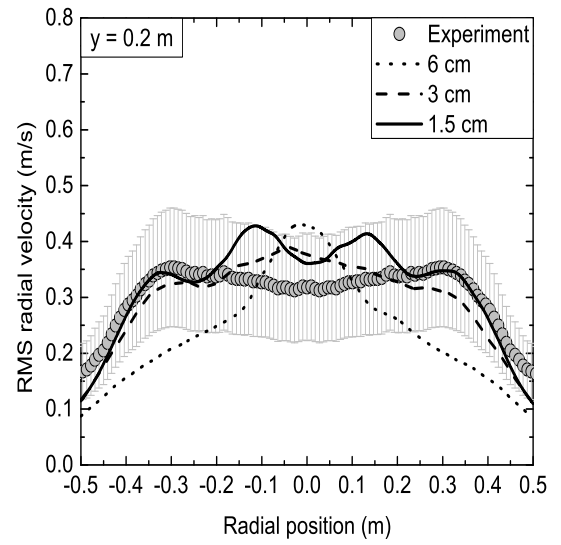

(b)

Figure 3: Radial profiles of (a) mean and (b) rms quantities at height $y=0.2 \mathrm{~m}$. 

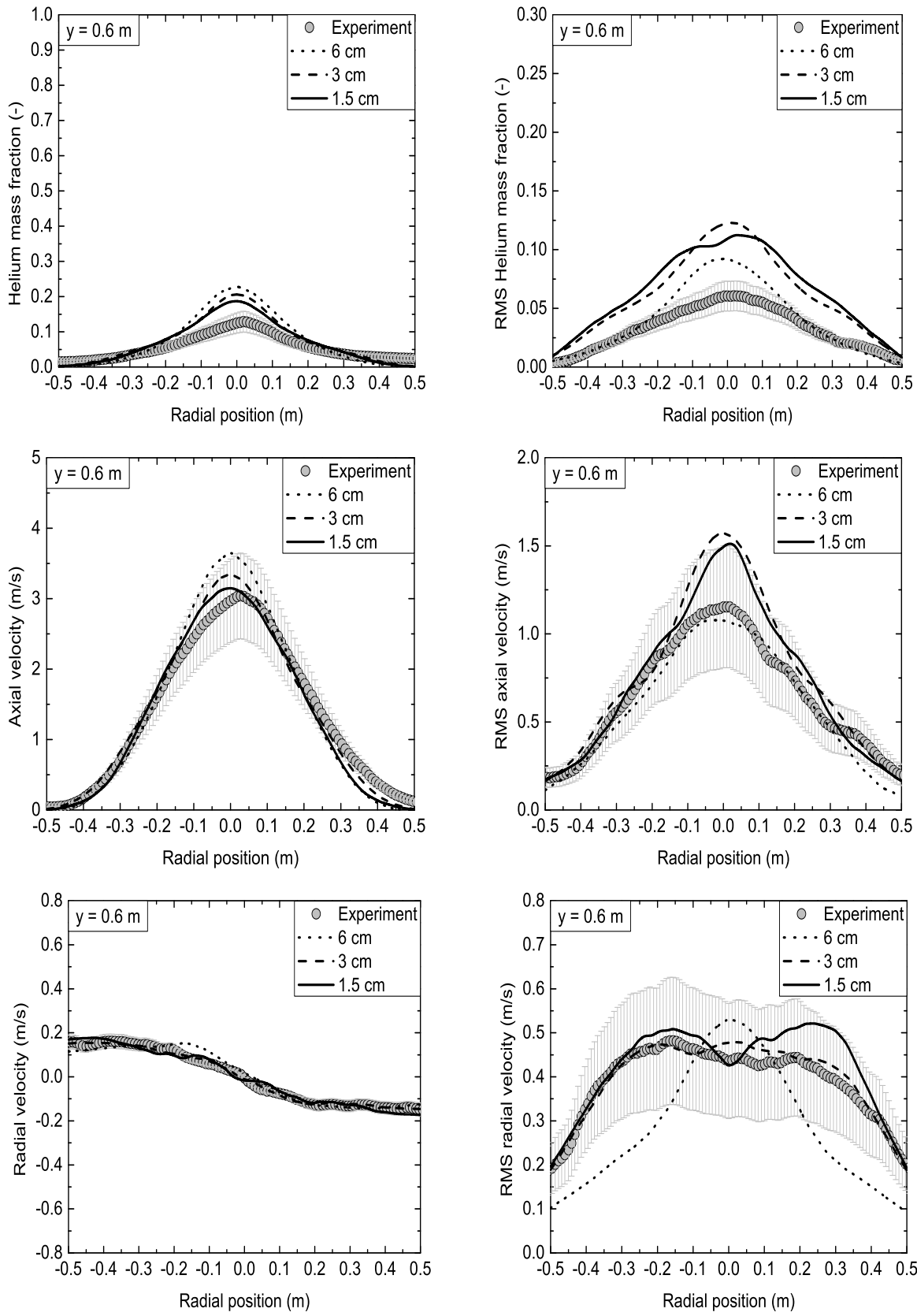

(a)

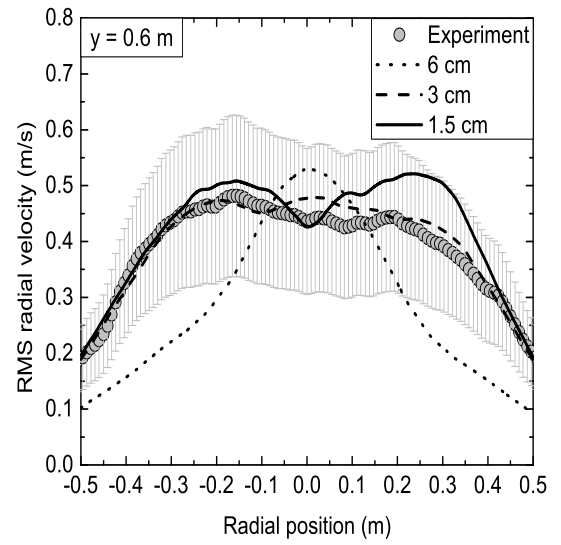

(b)

Figure 4: Radial profiles of (a) mean and (b) rms quantities at height $y=0.6 \mathrm{~m}$. 


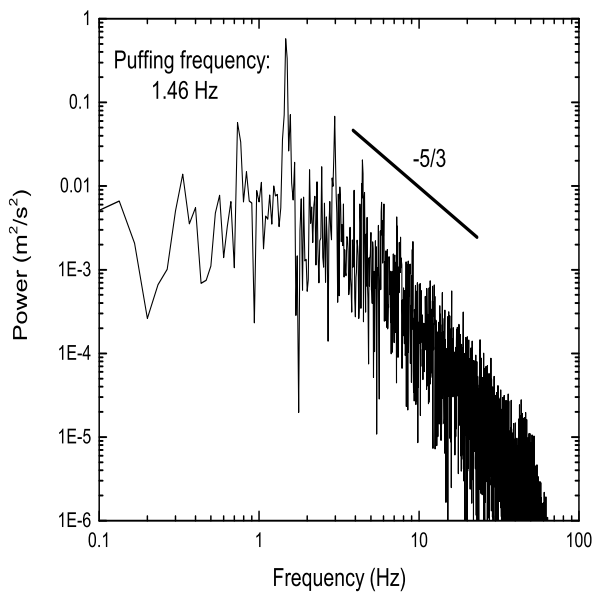

(a)

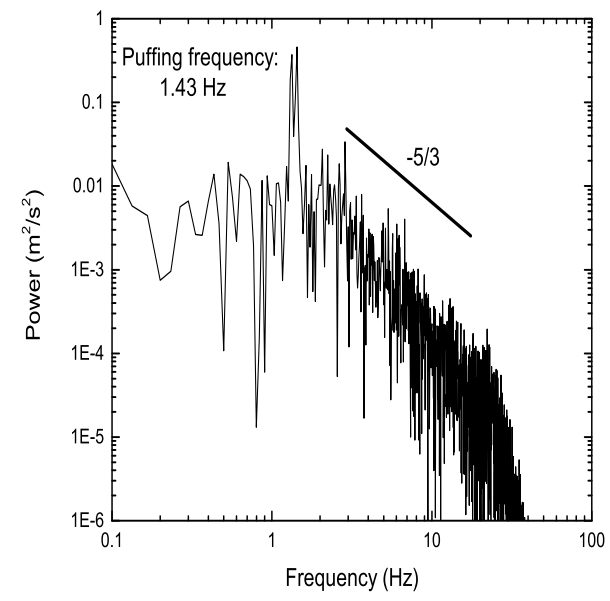

(b)

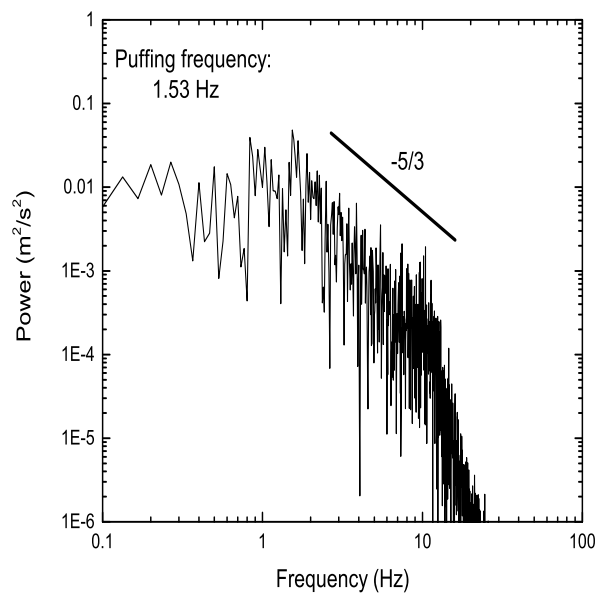

(c)

Figure 5: Predicted puffing frequency on the centerline at height $y=0.5 \mathrm{~m}$ with grid sizes of (a) $1.5 \mathrm{~cm}$, (b) $3 \mathrm{~cm}$ and (c) $6 \mathrm{~cm}$.

cells across the burner, a very coarse grid to expect reasonable predictions, even for simplified engineering calculations.

\subsection{UMD line burner}

The predicted radial profiles of mean temperatures at two different heights (with $X_{O 2}=0.18$ in the oxidizer stream), along with the predicted centerline mean and rms temperatures (with $X_{O 2}=0.21$ in the oxidizer stream), are 

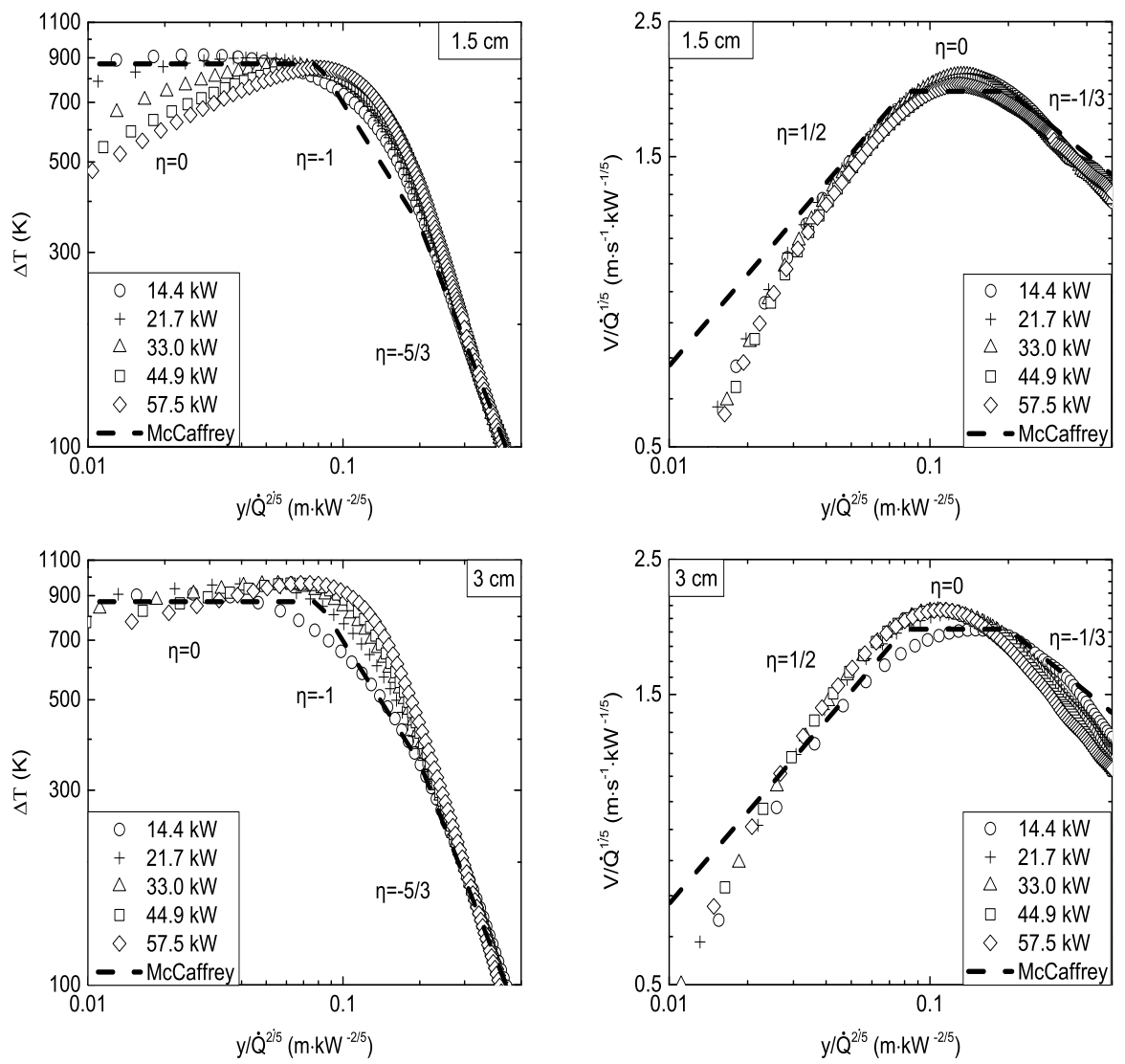

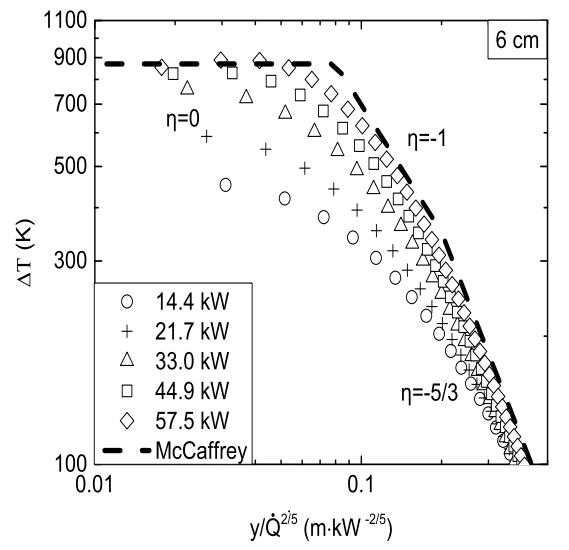

(a)

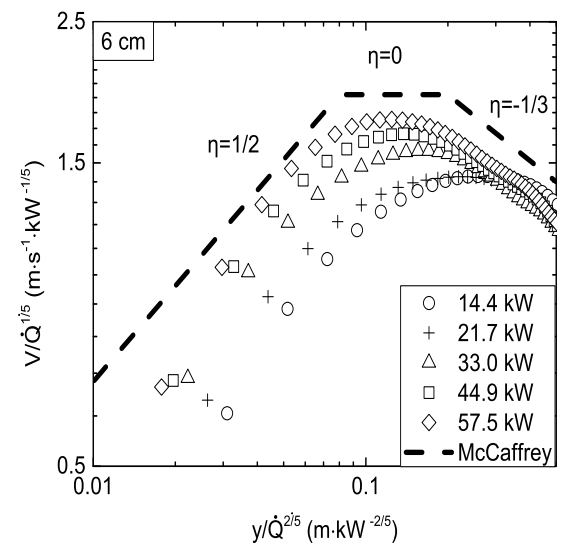

(b)

Figure 6: Centerline mean (a) temperatures and (b) axial velocities for different grid sizes. 
presented in Figure 7. It should be noted that in the case with $X_{O 2}=$ 0.18 the experimentally measured combustion efficiency was about $99 \%$, so no extinction modelling was considered. Overall, the numerical simulations on the finest grid size (i.e., $6.25 \mathrm{~mm}$ ) reproduce reasonably well both the temperature profiles at the two different locations examined as well as the mean and rms temperatures on the centerline. It is worth mentioning that the grid sizes of $25 \mathrm{~mm}, 12.5 \mathrm{~mm}$ and $6.25 \mathrm{~mm}$ employed in the simulations result in 2, 4 and 8 cells across the width (i.e., shortest side) of the burner, respectively. Only the latter case could perhaps be considered sufficient (i.e., minimum requirement), in terms of grid resolution, to simulate this scenario. Surprisingly enough, the first two cases also produce reasonable predictions in terms of resulting flame temperatures.

The experimentally reported radiative fractions were in the order of $19 \%$ and $24 \%$ for $X_{O 2}=0.18$ and $X_{O 2}=0.21$ in the oxidizer stream, respectively. The predicted radiative fractions for the case with $X_{O 2}=0.18$ in the oxidizer stream were $14.1 \%, 16.5 \%$ and $17.7 \%$ for the grid sizes of $25 \mathrm{~mm}, 12.5 \mathrm{~mm}$ and $6.25 \mathrm{~mm}$, respectively. The predicted radiative fractions for the case with $X_{O 2}=0.21$ in the oxidizer stream were $15.8 \%, 18.4 \%$ and $19.7 \%$ for the grid sizes of $25 \mathrm{~mm}, 12.5 \mathrm{~mm}$ and $6.25 \mathrm{~mm}$, respectively. It is evident that only the smallest grid size employed in the numerical simulations is fine enough to accurately resolve the flame brush, which will result in higher temperatures with the correct amount of heat being released due to radiation (i.e., correct predicted radiative fractions). It has been supported in the past that consideration of turbulence-radiation interactions (TRI) in turbulent flames can increase the radiative emissions through increased temperature fluctuations [34]. Nevertheless, TRI may be significantly less important for LES with sufficiently fine grids than in RANS [35]. It is believed that the discrepancies in the predicted radiative fractions with the two coarsest grid sizes (i.e., $25 \mathrm{~mm}$ and $12.5 \mathrm{~mm}$ ) are mainly due to insufficient local mixing but could be partly attributed to the neglection of TRI.

\subsection{Methanol pool fire}

Figure 8 presents the centerline mean and rms temperatures and axial velocities. The experimental data of Hamins et al. [36] of a methanol pool fire of the same diameter are also presented for comparison purposes. Overall, the fire plume does not seem to be turbulent enough near the pool fire surface for any of the grid sizes employed. It is postulated that the consideration of liquid evaporation could result in higher turbulence near the pool fire 


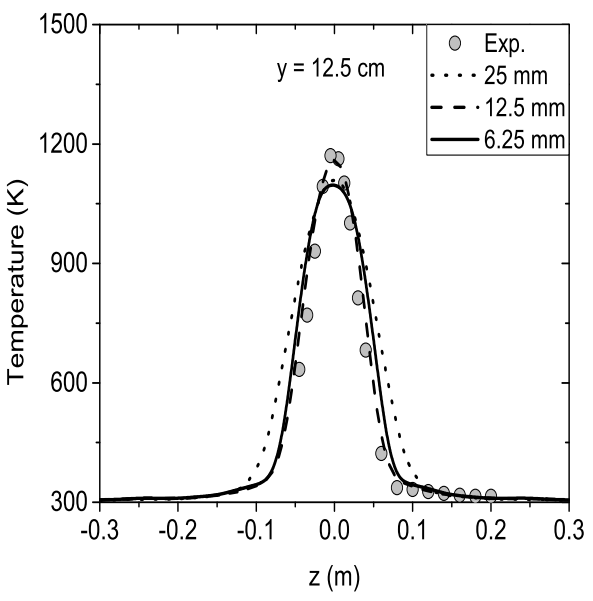

(a)

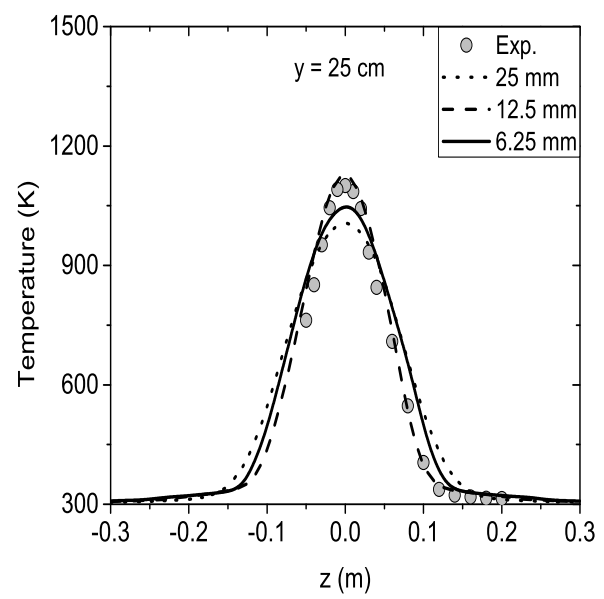

(b)

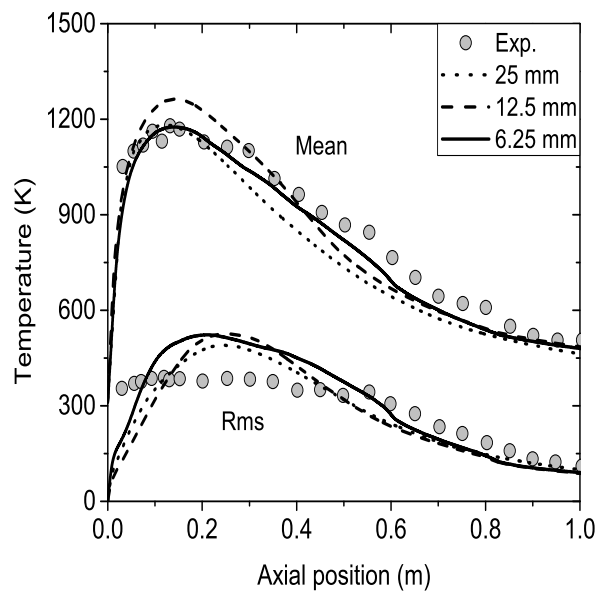

(c)

Figure 7: Predicted mean temperatures at height (a) $y=12.5 \mathrm{~cm}$ and (b) $y=25 \mathrm{~cm}$ when $X_{O 2}=0.18$ in the oxidizer stream while (c) depicts the predicted centerline mean and rms temperatures when $X_{O 2}=0.21$ in the oxidizer stream.

source and potentially improve the current predictions. The results with the $2 \mathrm{~cm}$ and $1 \mathrm{~cm}$ seem to be grid converged, but this is not confirmed for the $0.5 \mathrm{~cm}$ grid size. There is certainly some effect from the burner lip in this case, which alters the flow field close to the burner edges where the flame remains attached. In addition, it is not clear whether the consideration of the evaporation temperature of methanol at the pool fire source is an accurate 
assumption. There will be a very steep temperature gradient just above the surface, which the current grid size employed in the simulations can obviously not capture. Given the aforementioned uncertainties, the mean and rms predictions with all the grid sizes are fair and capture the overall trends of the experimental data, even though discrepancies are present.

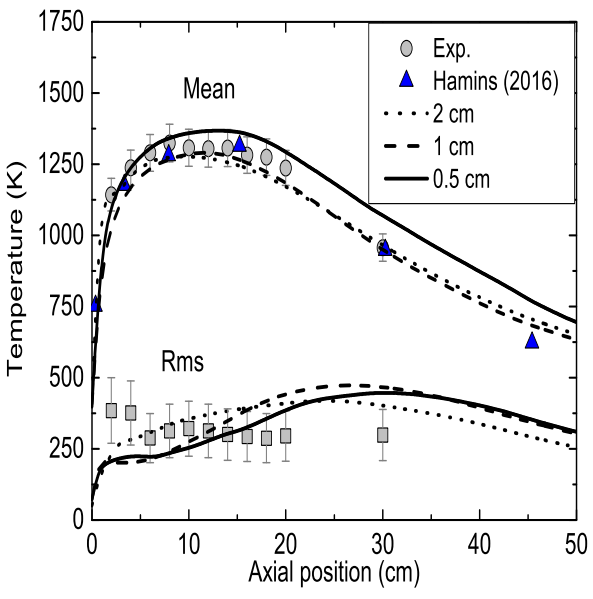

(a)

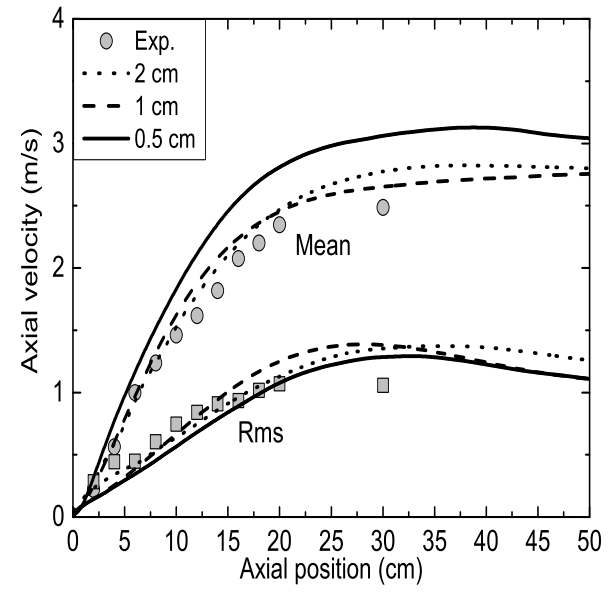

(b)

Figure 8: Predicted centerline mean and rms (a) temperatures and (b) axial velocities.

Radiative fractions for $30 \mathrm{~cm}$ methanol pool fires have been reported to be $18 \% \pm 26 \%$ [37], $18 \% \pm 10 \%$ [38], 17\% [39] and 22\% [40]. The numerical predictions were in the order of $14.6 \%, 17 \%$ and $17.5 \%$ for the $2 \mathrm{~cm}, 1 \mathrm{~cm}$ and $0.5 \mathrm{~cm}$ grid sizes, respectively, with the two finest grid resolutions reproducing fairly accurately the amount of heat being released due to radiation.

\section{Comparison to standard model}

In this section, numerical predictions are presented with the constant Smagorinsky model $\left(c_{s}=0.17\right.$ and $\left.S c_{t}=P r_{t}=0.5\right)$ for all the test cases considered in the study (Figures 10-11). The main objective is to assess whether the dynamic procedure previously employed for evaluating the turbulence model parameters, has greater advantages and leads to in improved predictions, compared to using models with constant parameters. To avoid repetition, only selected results are presented here in order to highlight the differences in the predictions between the two model approaches. 

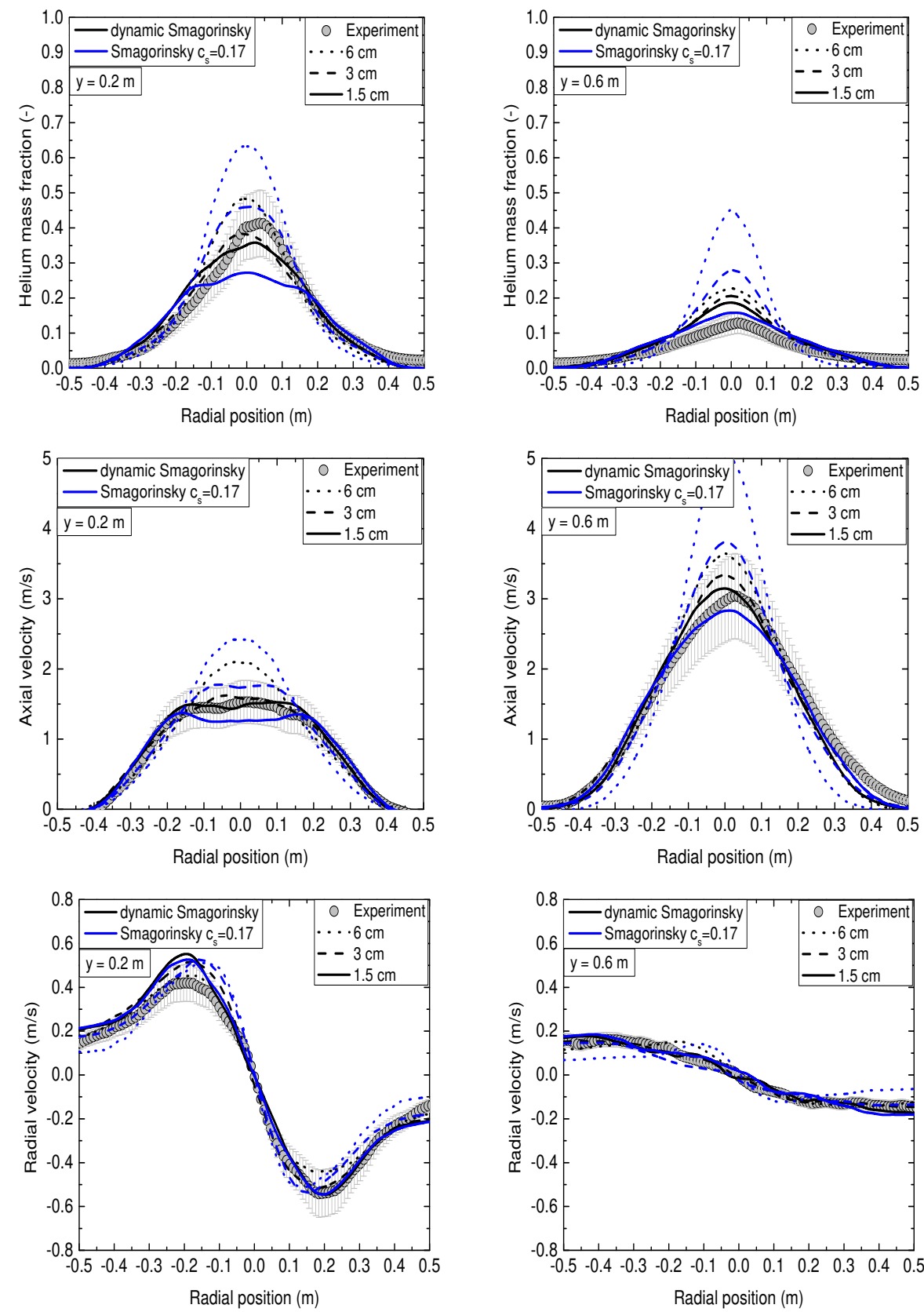

(a)

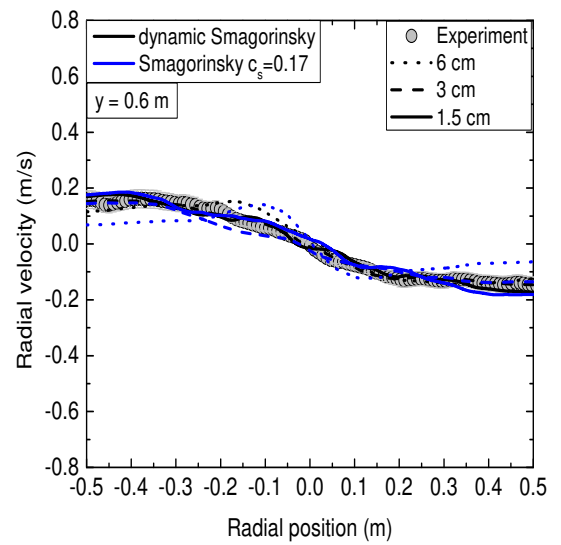

(b)

Figure 9: Radial profiles of mean quantities at height (a) $y=0.2 \mathrm{~m}$ and (b) $y=0.6 \mathrm{~m}$ with the dynamic and constant Smagorinsky model $\left(c_{s}=0.17\right)$ as a function of grid size for the Sandia helium plume case. 
The results for the helium plume case, shown in Figure 9, reveal a strong grid dependency when the constant Smagorinsky model is applied, with the predictions in terms of predicted helium mass fractions and axial velocities on the coarsest grid (i.e., $6 \mathrm{~cm}$ ) being relatively poor. The fact that the model is not able to self-adjust its model parameter, results in a flow that is not turbulent enough to break up the plume near the base of the helium source. Decreasing the model constant $c_{s}$ to values lower than 0.17 , in order to make the plume more turbulent, would most likely improve the predictions similarly to what has been previously reported in literature (e.g., [14]). Nevertheless, the choice of the model constant, unless chosen arbitrarily, would normally have to be based on an extensive sensitivity study and will be strongly case dependent. The results of the McCaffrey's case are presented in Figure 10 for the finest grid size employed in the simulations (i.e., $1.5 \mathrm{~cm}$ ). The scaling of the centerline excess temperature and velocity with the constant Smagorinsky model is not satisfactory when compared to the results with the dynamic model previously presented in Figure 6 . The numerical predictions are significantly over-predicted at locations $y / \dot{Q}^{2 / 5}>0.1$ (i.e., intermittent and plume regions) due to the absence of turbulence, indicating that the theoretical constant of $c_{s}=0.17$ is too high for this case. Similar observations can be made for the predicted centerline temperatures with the constant Smagorinsky model for the UMD line burner and methanol pool fires cases presented in Figures 11 and 12, respectively. The predictions with the theoretical value of $c_{s}=0.17$ do not agree favorably with the experimental data and are more grid sensitive than the prediction with the dynamic Smagorinsky model. In both cases, the theoretical constant seems to be too dissipative, essentially making the fire plumes less turbulent than needed.

From the results presented above and the $c_{s}$ and $c_{I}$ values previously reported in Figure 2, it is clear that the conditions near the base of most buoyant plumes examined here are laminar and it is essential to capture the laminar to turbulence transition in order to accurately simulate the different scenarios. Failing to do so, by e.g., using constant coefficients models with their parameters derived based on highly turbulent flows, results in more laminar-like structures (i.e., excessive turbulent dissipation) which affects the local mixing and the resulting plume dynamics. 


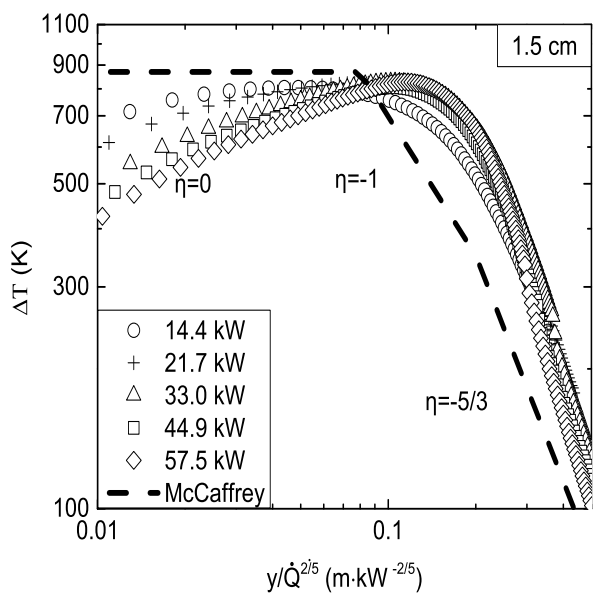

(a)

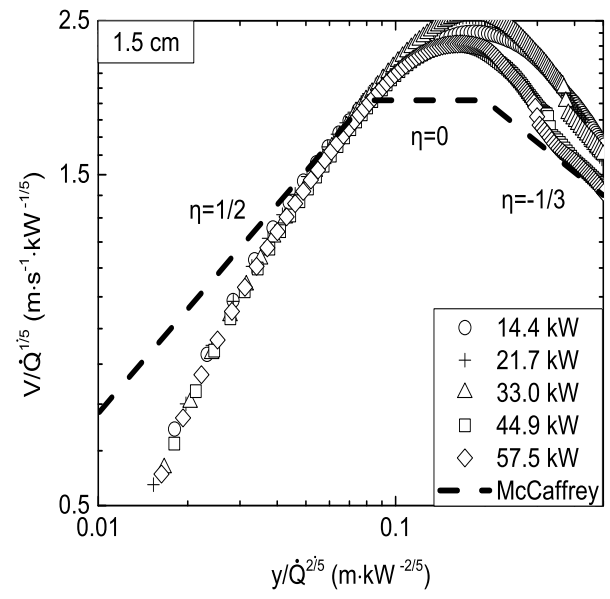

(b)

Figure 10: Centerline mean (a) temperatures and (b) axial velocities using the constant Smagorinsky model $\left(c_{s}=0.17\right)$ with a grid size of $1.5 \mathrm{~cm}$ for the McCaffrey fire plumes.

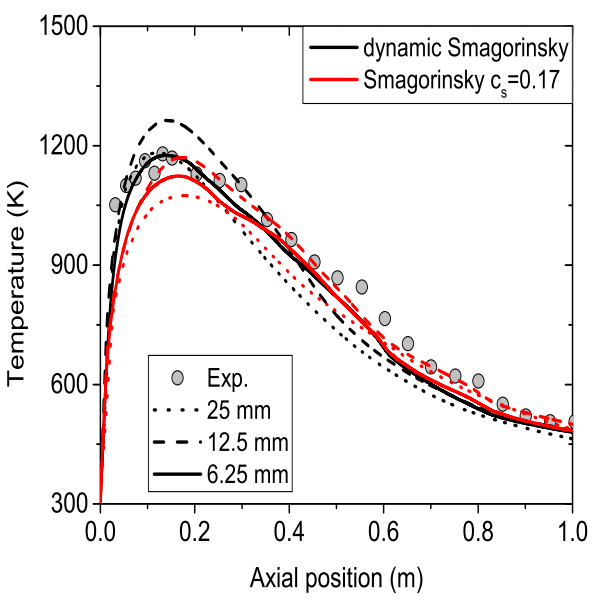

(a)

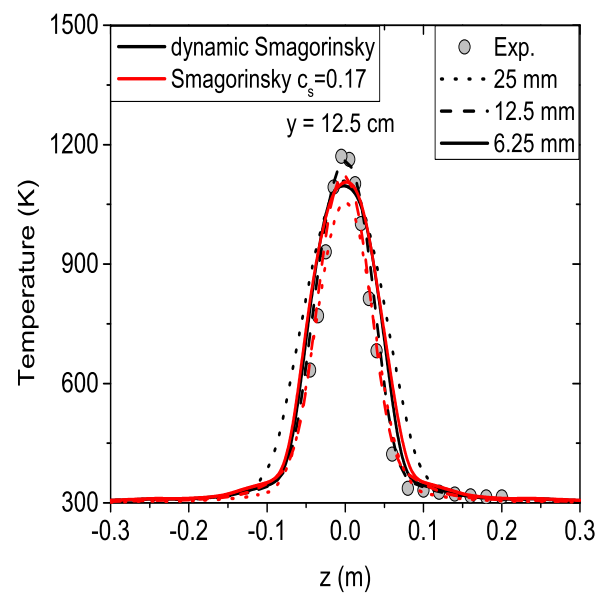

(b)

Figure 11: Mean temperatures (a) on the centerline and (b) at height $y=12.5 \mathrm{~cm}$ with the dynamic and constant Smagorinsky model $\left(c_{s}=0.17\right)$ as a function of grid size for the UMD line burner case. 


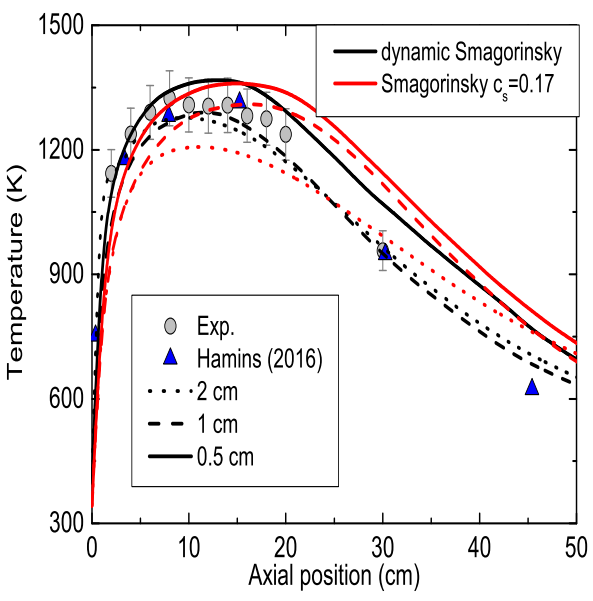

(a)

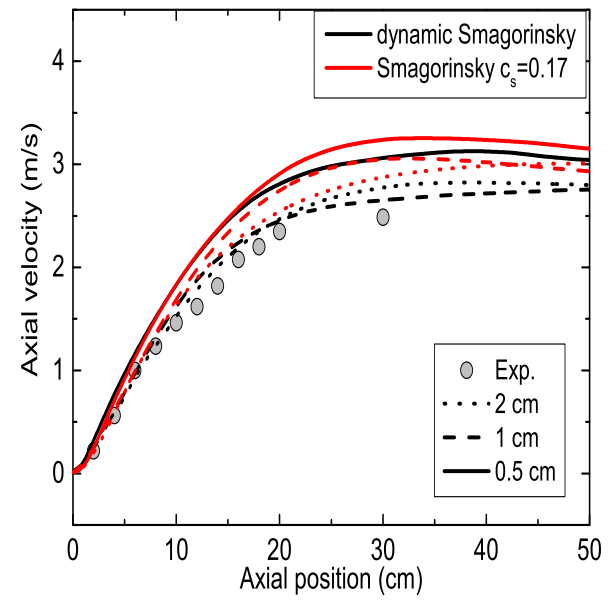

(b)

Figure 12: Centerline mean (a) temperatures and (b) axial velocities with the dynamic and constant Smagorinsky model $\left(c_{s}=0.17\right)$ as a function of grid size for the methanol pool fire case.

\section{Conclusions}

Large eddy simulations using dynamic modelling approaches have been performed and their predictive capabilities have been evaluated for a range buoyant plumes (i.e., MaCFP workshop test cases). The test cases considered for validation purposes consisted of the Sandia helium plume case, McCaffrey's fire plume experiments, UMD line burner and Waterloo's methanol pool fire. The main conclusions of the study are summarized as follows:

- Overall, the dynamic turbulence modelling approaches were capable of reproducing accurately the pool fire dynamics when comparing the resulting flame temperatures, axial and radial velocities, puffing frequencies and radiative fractions if grid sizes in the order of $1 \mathrm{~cm}$ were employed (i.e., $D^{*} / \Delta_{x}>10$ ). Both first and second order statistics have been discussed where possible. One of the main advantages of the current modelling approaches is that no calibration of any kind was needed/performed. The predictions with the standard Smagorinsky model (i.e., $c_{s}=0.17$ ) exhibited stronger grid dependency and were less accurate, compared to the dynamic version of the model, due to the fire plumes being less turbulent which affected local mixing, indi- 
cating that the theoretical model constant might be too dissipative for fire applications (which are typically not so strongly turbulent).

- The predicted turbulence model parameters related to sub-grid scale viscosity and kinetic energy, determined based on a dynamic procedure, were found to be significantly lower in all the plume scenarios examined, compared to their theoretical values obtained from homogeneous isotropic decaying turbulence. The dynamic procedure was illustrated to reproduce the values for isotropic decaying turbulence with the numerical scheme employed. More specifically, the resulting turbulence modelling parameters in the near-field $\left(y / L_{f}<0.2\right)$ region of the fire plumes were found to be range between $c_{s} \approx 0-0.1, c_{I} \approx 0.05-0.15$ and $\mathrm{Pr}_{t} / S c_{t} \approx 0.1-0.5$. The resulting average turbulence modelling parameters in the far-field region $\left(y / L_{f}>0.5\right)$ of the fire plumes were found to be in the order of $c_{s} \approx 0.1, c_{I} \approx 0.15$ and $P r_{t} / S c_{t} \approx 0.5$. Nevertheless, significant variations around these values were evident depending on the location examined and the dynamic procedure was able to capture them to some extent.

The present work aimed at indicating the potential of using dynamic modelling approaches in order to enhance predictive fire modelling. Further evaluation of these models in more challenging fire scenarios involving e.g., flame spread and pyrolysis, would be interesting to investigate in the future.

\section{Acknowledgments}

This research has been funded by Ghent University (Belgium) through GOA project BOF16/GOA/004.

\section{References}

[1] J. Smagorinsky, General circulation experiments with the primitive equations: I. The basic experiment, Mon Weather Rev. 91 (1963) 99-164.

[2] B. Vreman, An eddy-viscosity subgrid-scale model for turbulent shear flow: Algebraic theory and applications, Phys. Fluids 16 (2004) 36703681. 
[3] U. Schumann, Subgrid scale model for finite difference simulations of turbulent flows in plane channels and annuli, J. Comput. Phys. 18 (1975) 376-404.

[4] M. Germano, U. Piomelli, P. Moin, W.H. Cabot, A dynamic subgridscale eddy viscosity model, Phys. Fluids A 3, 1760-1765 (1991).

[5] D.K. Lilly, A proposed modification of the germano subgrid-scale closure model, Phys. Fluids A 4, 633-635 (1992).

[6] C. Fureby, G. Tabor, Mathematical and Physical Constrains on LargeEddy Simulations, Theoret. Comput. Fluid Dynamics, 9 (1997) 85-102.

[7] M.P. Martin, U. Piomelli, G.V. Candler, Sub-grid-Scale Models for Compressible Large-Eddy Simulations, Theoret. Comput. Fluid Dynamics 13 (2000) 361-376.

[8] C. Meneveau, T.S. Lund, The dynamic Smagorinsky model and scaledependent coefficients in the viscous range of turbulence, Phys. Fluids 9 (1997) 3932-3934.

[9] J. Meyers, P. Sagaut, On the model coefficients for the standard and the variational multi-scale Smagorinsky model, J. Fluid Mech. 569 (2006) 287-319.

[10] P.E. DesJardin, T.J. O'Hern, S.R. Tieszen, Large eddy simulation and experimental measurements of the near-field of a large turbulent helium plume, Phys. Fluids 16 (2004) 1866-1883.

[11] P.E. DesJardin, Modeling of conditional dissipation rate for flamelet models with application to large eddy simulation of fire plumes, Combust. Sci. Technol. 177 (2005) 1883-1916.

[12] A. Brown, M. Bruns, M. Gollner, J. Hewson, G. Maragkos, A. Marshall, R. McDermott, B. Merci, T. Rogaume, S. Stoliarov, J. Torero, A. Trouvé, Y. Wang, E. Weckman, Proceedings of the first workshop organized by the IAFSS Working Group on Measurement and Computation of Fire Phenomena (MaCFP), Fire Saf. J. 101 (2018) 1-17.

[13] G. Maragkos, P. Rauwoens, B. Merci, Application of FDS and FireFOAM in Large Eddy Simulations of a Turbulent Buoyant Helium Plume, Combust. Sci. Technol. 184 (2012) 1108-1120. 
[14] G. Maragkos, P. Rauwoens, Y. Wang, B. Merci, Large Eddy Simulations of the Flow in the Near-Field Region of a Turbulent Buoyant Helium Plume, Flow Turbul. Combust. 90 (2013) 511-543.

[15] G. Maragkos, T. Beji, B. Merci, Advances in modelling in CFD simulations of turbulent gaseous pool fires, Combust. Flame 181 (2017) 22-38.

[16] G. Maragkos, B. Merci, Large eddy simulations of $\mathrm{CH}_{4}$ fire plumes, Flow Turb. Combust. 99 (2017) 239-278.

[17] G. Maragkos, T. Beji, B. Merci, Towards predictive simulations of gaseous pool fires, Proc. Combust. Inst. 37 (2019) 3927-3934.

[18] https://github.com/fireFoam-dev

[19] P. Moin, K. Squires, W.H. Cabot, S. Lee, A dynamic subgrid-scale model for compressible turbulence and scalar transport, Phys. Fluids A 3 (1991) 2746-2757.

[20] T.S. Lund, On the use of discrete filters for large eddy simulation, Annual Research Briefs, Center for Turbulence Research, NASA Ames/Stanford University (1997) 83-95.

[21] S. Menon, W.-W. Kim, High Reynolds number flow simulations using the localized dynamic subgrid-scale model, 34th Aerospace Sciences Meeting and Exhibit, Aerospace Sciences Meetings, 1996.

[22] B.F. Magnussen, B.H. Hjertager, On Mathematical Modeling of Turbulent Combustion with Special Emphasis on Soot Formation and Combustion, Proc. Comb. Inst. 16 (1977) 719-729.

[23] E.A. Brizuela, R.W. Bilger, On the eddy break-up coefficient, Combust. Flame 104 (1996) 208-212.

[24] L.J. Dorigon, G. Duciak, R. Brittes, F. Cassol, M. Galarca, F.H.R. Franca, Int. J. Heat Mass Transf. 64 (2013) 863-873.

[25] T.J. O'Hern, E.J. Weckman, A.L. Gerhart, S.R. Tieszen, R.W. Schefer, Experimental study of a turbulent buoyant helium plume, J. Fluid Mech. 544 (2005) 143-171. 
[26] B.J. McCaffrey, Purely Buoyant Diffusion Flames: Some Experimental Results, National Bureau of Standards, NBSIR 79-1910, 1979.

[27] J.P. White, E.D. Link, A.C. Trouvé, P.B. Sunderland, A.W. Marshall, J.A. Sheffel, M.L. Corn, M.B. Colket, M. Chaos, H.-Z. Yu., Radiative emissions measurements from a buoyant, turbulent line flame under oxidizer-dilution quenching conditions, Fire Saf. J. 76 (2015) 74-84.

[28] E.J. Weckman, A.B. Strong, Experimental Investigation of the Turbulence Structure of Medium-Scale Methanol Pool Fires, Combust. Flame 105 (1996) 245-266.

[29] K. McGrattan, S. Hostikka, R. McDermott, J. Floyd, M. Vanella, C. Weinschenk, K. Overholt, Fire Dynamics Simulator Users Guide, NIST Special Publication 1019, Sixth Edition, 2017.

[30] B.J. McCaffrey, Some Measurements of the Radiative Power Output of Diffusion Flames, Proceedings of the Western States Section of the Combustion Institute, 1981.

[31] N. Ren, Y. Wang, S. Vilfayeau, A. Trouvé, Large eddy simulation of turbulent vertical wall fires supplied with gaseous fuel through porous burners, Combust. Flame 169 (2016) 194-208.

[32] B.M. Cetegen, K.D. Kasper, Experiments on the oscillatory behaviour of buoyant plumes of helium and helium-air mixtures, Phys. Fluids 8 (1996) 2974-2984.

[33] B.M. Cetegen, T.A. Ahmed, Experiments on the periodic instability of buoyant plumes and pool fires, Combust. Flame 93 (1993) 157-184.

[34] G. Cox, On Radiant Heat Transfer from Turbulent Flames, Combust. Sci. Technol. 17 (1977) 7578.

[35] F.C. Miranda, P.J.Coelho, F. di Mare, J. Janicka,Study of turbulenceradiation interactions in large-eddy simulation of scaled Sandia flame D, J. Quant. Spectrosc. Radiat. Transf. 228 (2019) 47-56.

[36] A.P. Hamins, A. Lock, The Structure of a Moderate-Scale Methanol Pool Fire, Technical Note (NIST TN) - 1928, 2016. 
[37] S.C. Kim, K.Y. Lee, A. Hamins, Energy Balance in Medium-Scale Methanol, Ethanol, and Acetone Pool Fires, Fire Saf. J. (2019) - In Press.

[38] A. Hamins, M. Klassen, J. Gore, T. Kashiwagi, Estimate of Flame Radiance via a Single Location Measurement in Liquid Pool Fires, Combust. Flame 86 (1991) 223-228.

[39] A. Hamins, M. Klassen, J. Gore, S. Fischer, T. Kashiwagi, Heat Feedback to the Fuel Surface in Pool Fires, Combust. Sci. Tech. 97 (1993) $37-62$.

[40] R. Buch, A. Hamins, K. Konishi, D. Mattingly, T. Kashiwagi, Radiative Emission Fraction of Pool Fires Burning Silicone Fluids, Combust. Flame 108 (1997) 118-126.

[41] S.B. Pope, Turbulent Flows, Cambridge University Press, 2000.

[42] C. Fureby, G. Tabor, H.G. Weller, A.D. Gosman, A comparative study of subgrid scale models in homogeneous isotropic turbulence, Phys. Fluids 9 (1997) 1416-1429.

[43] J.W. Deardorff, The use of subgrid transport equations in a threedimensional model of atmospheric turbulence, J. Fluids Eng. 95 (1973) 429-438.

[44] G. Comte-Bellot, S. Corrsin, Simple Eulerian time correlation of full-and narrow-band velocity signals in grid-generated, 'isotropic' turbulence, J. Fluid Mech., 48 (1971) 273-337.

[45] K. McGrattan, S. Hostikka, R. McDermott, J. Floyd, M. Vanella, Fire Dynamics Simulator Technical Reference Guide Volume 2: Verification, NIST Special Publication 1018-2, Sixth Edition, 2018.

[46] https://github.com/firemodels/fds/tree/master/Verification/Turbulence

[47] T.M. Eidson, Numerical simulation of the turbulent Rayleigh-Bénard problem using subgrid model, J. Fluid Mech. 158 (1985) 245-268.

[48] J. Craske1, P. Salizzoni, M. Reeuwijk, The turbulent Prandtl number in a pure plume is 3/5, J. Fluid Mech. 822 (2017) 774790. 
[49] M.V. Pham, F. Plourde, K.S. Doan, Direct and large-eddy simulations of a pure thermal plume, Phys. Fluids 19 (2007) 125103.

[50] J. Lei, N. Kiu, Scaling flame height of fully turbulent pool fires based on the turbulent transport properties, Proc. Comb. Inst. 36 (2017) 31393148 . 